\title{
Apofonia e o sistema vocálico do Proto-Jê Meridional: contribuição para estudos comparativos das línguas $\mathbf{J} \hat{e}^{1}$
}

\author{
Apophony and vocalism of Proto-Southern Jê: \\ a contribution to comparative Jê studies
}

Andrey Nikulin ${ }^{2}$

\begin{abstract}
Resumo
A reconstrução fonológica da protolíngua de Kaingáng, Kaingáng Paulista, Xokléng e Ingain (todas da região Sul do Brasil e áreas adjacentes) já foi abordada por vários pesquisadores. Este trabalho visa a reavaliar as decisões tomadas previamente em relação à reconstrução do sistema vocálico desta protolíngua, baseando-se em algumas idiossincrasias registradas sincronicamente nas línguas Kaingáng e Xokléng, assim como na comparação externa. Demonstra-se, por exemplo, que o Proto-Jê Meridional possuía dez vogais orais (e não nove). A análise de vários empréstimos da língua Mbyá na língua Kaingáng corrobora a hipótese. É apresentada ainda uma proposta de reconstrução do sistema vocálico do ProtoJê, assim como as correspondências das vogais do Proto-Jê com o Maxakalí e o ProtoJabutí.
\end{abstract}

Palavras-chave: Fonologia histórica. Reconstrução linguística. Línguas Jê. Línguas MacroJê. Línguas Jê Meridionais.

\begin{abstract}
The phonological reconstruction of the proto-language of Kaingáng, São Paulo Kaingáng, Xokléng and Ingain (all spoken in the Southern region of Brazil) has already been attempted by a variety of scholars. This work aims to reevaluate the decisions that have been previously made concerning the reconstruction of the vocalism of the proto-language in question, taking into account some idiosyncrasies attested synchronically in Kaingáng and Xokléng as well as external comparison. It is also demonstrated that the vocalism of Proto-Southern Jê comprised ten oral vowels (as opposed to nine). The analysis of Mbyá borrowings in Kaingáng corroborates the hypothesis. A reconstruction of the Proto-Jê vocalism is also attempted. The reconstructed vowel phonemes are further compared to Maxakalí and ProtoJabutí vowels.
\end{abstract}

Keywords: Historical phonology. Language reconstruction. Jê languages. Macro-Jê languages. Southern Jê languages.

\footnotetext{
${ }^{1}$ Agradecimentos a Pedro Taam pelo trabalho de revisão.

${ }^{2}$ Universidade de Brasília; Academia de Ciências da Rússia (Instituto de Estudos Orientais).
} 


\section{Introdução}

O ramo Jê Meridional pertencente à família Jê do tronco linguístico Macro-Jê é constituído de quatro línguas, a saber: Kaingáng, Kaingáng Paulista, Xokléng e Ingain/Kimdá3. Reconstruções fonológicas da protolíngua deste ramo (daqui em diante referida como PJM) foram previamente propostas por Wiesemann (1958, 1978), D’Angelis (2007-2008, 2009) e Jolkesky (2010). Este último apresenta também um vocabulário comparado com mais de mil protoformas reconstruídas.

O ramo em questão constitui, junto com o ramo Jê Amazônico, a família linguística Jê. O ramo Jê Amazônico inclui dois ramos bem estabelecidos: o Jê Norocidental e o Jê Central (D’Angelis 2009:134, Nikulin 2015:11). Essa classificação é comprovada pela presença de inovações fonológicas e lexicais comuns em todos os ramos acima mencionados. Aplicando-se o método léxicoestatístico aperfeiçoado por Starostin (2010) e Kassian et al. (2010), obteve-se o mesmo modelo (Nikulin 2016, no prelo). Alguns autores, porém, dividem a família Jê diretamente em três ramos, sem recorrer à noção do ramo Jê Amazônico, a saber: Jê Meridional, Jê Central e Jê Setentrional (Rodrigues 2012:284).

O presente artigo visa a reconsiderar a reconstrução do sistema vocálico do PJM, baseando-se em algumas idiossincrasias morfofonológicas registradas sincronicamente em Kaingáng e Xokléng, assim como na comparação externa com e em considerações sistêmicas.

\section{Trabalhos anteriores}

Jolkesky (2010:161) reconstrói o seguinte sistema fonológico para o PJM (notação do autor ${ }^{4}$ ):

Quadro 1: Protofonemas consonantais e vocálicos do PJM segundo Jolkesky (2010)

\begin{tabular}{|c|c|c|c|c|c|}
\hline \multicolumn{2}{|c|}{ CONSOANTES } & \multicolumn{2}{|c|}{$[+$ ant $]$} & \multicolumn{2}{c|}{$[$-ant] } \\
\cline { 3 - 6 } & {$[$-cor $]$} & {$[+$ cor $]$} & {$[+$ cor $]$} & {$[$-cor $]$} \\
\hline \multirow{2}{*}[\text{-son}]{} & {$[$-cont $]$} & $*_{p}$ & $*_{t}$ & $*_{c}$ & $*_{k}$ \\
\cline { 2 - 6 } & {$[+$ cont $]$} & \multicolumn{3}{|c|}{$*_{s}$} \\
\hline \multirow{2}{*}[+\text{son}]{} & {$[$-cont $]$} & $*_{b}$ & $*_{d}$ & $*_{f}$ & $*_{g}$ \\
\cline { 2 - 6 } & {$[+$ cont $]$} & $*_{w}$ & $*_{r}$ & $*_{j}$ & $*_{h}$ \\
\hline
\end{tabular}

${ }^{3}$ Daqui em diante, Kgg, KgP, Xok e Ing respectivamente. Em nossa opinião, Ingain e Kimdá são o mesmo idioma, já que praticamente todas as palavras do pequeno vocabulário Kimdá encontrado em (Lista 1883) são quase idênticas às palavras encontradas nos registros da língua Ingain.

${ }^{4}$ Vale a pena ressaltar que Jolkesky utiliza o modelo representacional da Geometria dos Traços. 


\begin{tabular}{|c|c|c|c|}
\hline \multirow{2}{*}{ VOGAIS ORAIS } & \multirow{2}{*}{-post] } & \multicolumn{2}{|c|}{$[+$ post] } \\
\cline { 3 - 4 } & $*_{i}$ & $*_{u}$ & $*_{u}$ \\
\hline [+alto] & $*_{e}$ & $*_{\gamma}$ & $*_{o}$ \\
\hline [-alto] \\
{$[$-baixo] } & $*_{\varepsilon}$ & $*_{a}$ & $*_{j}$ \\
\hline [+baixo] & &
\end{tabular}

\begin{tabular}{|c|c|c|c|}
\hline \multirow{2}{*}{ VOGAIS NASAIS } & \multirow{2}{*}[\text{-post}]{} & {$[+$ post $]$} & {$[$-post $]$} \\
\cline { 3 - 4 } & & {$[$-arred $]$} & {$[+$ arred $]$} \\
\hline$[+$ alto $]$ & $*_{\tilde{1}}$ & $* \tilde{\mathrm{u}}$ & $*_{\tilde{\mathrm{u}}}$ \\
\hline$[$-alto $]$ & $*_{\tilde{\varepsilon}}$ & $*_{\tilde{a}}$ & $* \tilde{\jmath}$ \\
\hline
\end{tabular}

Esses protofonemas teriam tido os seguintes reflexos nas línguas registradas:

Quadro 2: Reflexos dos protofonemas do PJM em Kaingáng, Kaingáng Paulista e Xokléng segundo Jolkesky (2010)

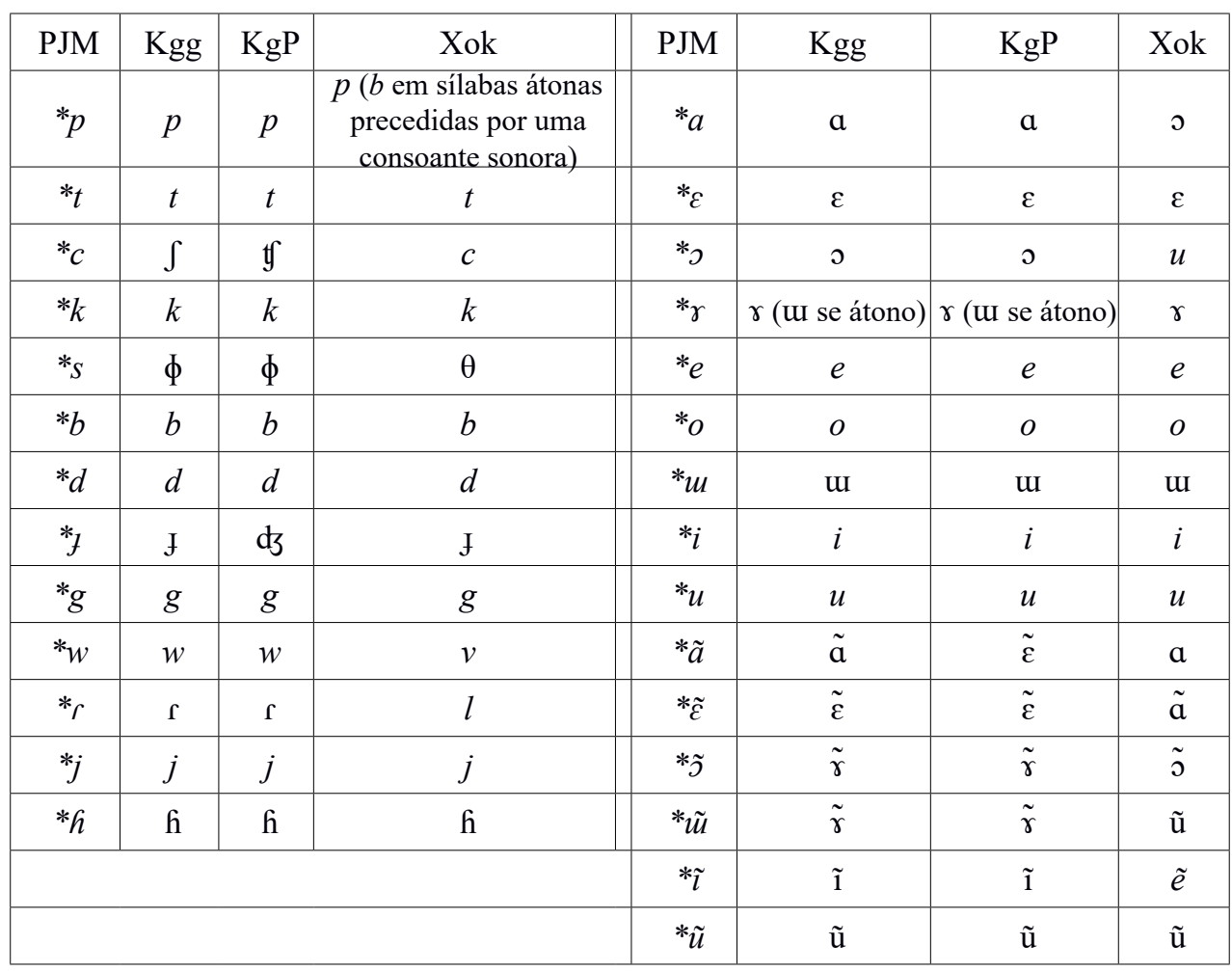

${ }^{5}$ Nós optamos por excluir a língua Ingain deste quadro porque muitas das suposições sobre a fonologia Ingain aceitas por Jolkesky nos parecem arbitrárias, o que impossibilita o uso da fonematização dos dados Ingain aceita por ele para a reconstrução fonológica do PJM. 
O onset da sílaba podia ser ocupado por qualquer das consoantes ou ainda por qualquer das sequências $* p r,{ }^{*} b r$, ${ }^{*} k r$ ou $* g r$. A coda podia ser ocupada por qualquer das consoantes sonoras, exceto $* h$.

As propostas anteriores de Wiesemann e D'Angelis (2007-2008, 2009) diferem, notavelmente, na interpretação fonética do protofonema contínuo surdo: Wiesemann (1958) e D'Angelis (2007-2008) reconstroem *f, enquanto em outro trabalho da própria Wiesemann (1978) a mesma correspondência é associada com o protofonema *ð. Além disso, D'Angelis reconstrói um protofonema glotal *? (cuja ocorrência é automática, já que o fonema em questão ocupa a posição do onset na ausência de outras consoantes na interpretação de Jolkesky).

Uma diferença maior entre as reconstruções de Jolkesky e D'Angelis é aquela que existe entre os cenários da evolução dos sistemas vocálicos que eles

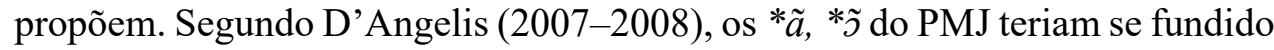
ainda na protolíngua (esses fonemas correspondem a *ã na reconstrução de Jolkesky). D’Angelis reconstrói *ã onde Jolkesky reconstrói *̃̃ e não identifica a correspondência entre $\tilde{\gamma}$ em Kaingáng e Kaingáng Paulista e $\tilde{u}$ em Xokléng. Ambos os autores concordam no que tange à reconstrução de um fonema nasal baixo para a correspondência Kaingáng $\tilde{a} \sim$ Kaingáng Paulista $\tilde{\varepsilon} \sim$ Xokléng $a$ e de um fonema oral baixo para a correspondência Kaingáng $a \sim$ Kaingáng Paulista $a \sim$ Xokléng $\curvearrowright$. Os autores não explicam por que consideram o Xokléng mais inovador nestes casos. Mostraremos na seção 5 que, na verdade, há motivos para considerar o Xokléng mais conservador.

\section{Alofonia consonantal nas línguas Jê Meridionais}

Parece-nos essencial levar sempre em conta as realizações fonéticas dos fonemas das línguas registradas, já que as leis fonéticas atingem fones e não fonemas. Por exemplo, apesar de as consoantes pré-nasalizadas e nasais terem sido alofones dos mesmos fonemas na língua Proto-Jê, os reflexos desses alofones são muito diferentes em várias línguas Jê, tais como Xavánte, Xerénte e Timbíra (Nikulin 2015:15-16).

Considerar os alofones em vez de fonemas é importante ainda porque em alguns casos os dados sincrônicos permitem mais de uma análise, ainda que nem todas elas sejam adequadas diacronicamente. Por exemplo, na língua Mundurukú os fones $\eta$ e $n$ ocorrem em distribuição complementar: $\eta$ é encontrado exclusivamente em coda silábica, enquanto $n$ só é encontrado na posição de onset. Na ortografia utilizada pelos Mundurukú os dois fones são representados pelo símbolo $\tilde{g}$; em obras linguísticas ambos são comumente denotados por $/ \eta /$. Entretanto, conforme mostra Picanço (2005:198), de um ponto de vista diacrônico, seria mais adequado interpretar $n$ como um alofone de $/ \check{\zeta} /$.

Os fonemas consonantais em todas as línguas Jê Meridionais são conhecidos 
por um grande número de possíveis realizações fonéticas. O fator principal que contribui para a variedade das realizações alofônicas dos fonemas consonantais em quase todas as línguas Jê (tanto Meridionais, quanto Amazônicas) ${ }^{6}$ é a nasalidade da vogal que forma o núcleo da sílaba (Jolkesky 2010:71-75, 9395, 107-109, Pickering 2010:62, Camargo 2010:70-74, Oliveira 2005:38-55). Diferentemente do que se observa em várias línguas dos troncos Tupí e Nadahup (Thomas 2014, Costa 2007, Epps 2005:72-75), a nasalidade nas línguas Jê não é uma característica suprassegmental e é claramente uma propriedade intrínseca das vogais e não de unidades maiores.

Estão apresentadas, a seguir, as correspondências entre os segmentos consonantais das línguas Kaingáng, Kaingáng Paulista e Xokléng para a posição de onset (Quadro 3) e coda (Quadro 4). Propomos uma reconstrução fonética para cada correspondência.

Quadro 3. Correspondências entre os fones consonantais das línguas Jê Meridionais registradas para a posição de onset ${ }^{7}$

\begin{tabular}{|c|c|c|c|c|c|c|c|}
\hline \multicolumn{2}{|c|}{$\begin{array}{c}\text { PJM } \\
\text { (proposta) }\end{array}$} & \multicolumn{2}{|c|}{$\mathrm{Kgg}$} & \multicolumn{2}{|c|}{$\mathrm{KgP}$} & \multicolumn{2}{|c|}{ Xok } \\
\hline _O & _N & _O & ${ }_{-} \mathrm{N}$ & _O & ${ }_{-} \mathrm{N}$ & ${ }_{-} \mathrm{O}$ & ${ }_{-} \mathrm{N}$ \\
\hline \multicolumn{2}{|c|}{${ }^{*} p$} & \multicolumn{2}{|c|}{$p$} & \multicolumn{2}{|c|}{$p$} & \multicolumn{2}{|c|}{$\begin{array}{l}p \text { ( }{ }^{n} b \text { em sílabas átonas } \\
\text { precedidas por uma } \\
\text { consoante sonora) }\end{array}$} \\
\hline \multicolumn{2}{|c|}{${ }^{*} t$} & \multicolumn{2}{|c|}{$t$} & \multicolumn{2}{|c|}{$t$} & \multicolumn{2}{|c|}{$\stackrel{t}{\sim}$} \\
\hline \multicolumn{2}{|c|}{${ }^{*} c$} & $c ̧ \sim \int$ & $\tilde{c} \overline{\bar{J}} \sim \sqrt{3}$ & \multicolumn{2}{|c|}{$\bar{t} \boldsymbol{t}$} & \multicolumn{2}{|c|}{$c \sim \widehat{t f}$} \\
\hline \multicolumn{2}{|c|}{$*_{k}$} & \multicolumn{2}{|c|}{$k$} & \multicolumn{2}{|c|}{$k$} & \multicolumn{2}{|c|}{$k$} \\
\hline
\end{tabular}

${ }^{6}$ Uma possível exceção seria a língua Xerénte, na qual a queda de vogais teria fonematizado a variação alofônica dos fonemas consonantais do Proto-Jê Central na posição de onset (Sousa 2008: 91-93, Grannier 2009), contra (Mattos 1973). Além disso, nos dialetos do contínuo dialetal Timbíra houve uma neutralização dos alofones pré-nasalizados dos fonemas sonoros nasais com os fonemas oclusivos surdos, deixando, no entanto, vestígios morfofonológicos deste contraste (Amado 2004: 75-76). O ensurdecimento dos alofones pré-nasalizados ocorreu também na língua Panará, gerando sequências de uma vogal alta posterior não-arredondada nasal e oclusivas surdas. A alofonia em questão deixou de existir no onset silábico em Kayapó, mas permaneceu na posição de coda (Stout \& Thomson 1974: 2), assim como em vários dialetos Timbíra, tais como Canela (Popjes \& Popjes 1971: 9).

${ }^{7}$ Abreviações: _O = seguido de vogal oral, $\_\mathrm{N}=$ seguido de vogal nasal, $\mathrm{O}_{-}=$depois de vogal oral antes de consoante sonora ou pausa, $\mathrm{N}_{-}=$depois de vogal nasal antes de consoante sonora ou pausa,_S = antes de consoante surda. 


\begin{tabular}{|c|c|c|c|c|c|c|c|}
\hline \multicolumn{2}{|c|}{$* 7^{8}$} & \multirow{2}{*}{$\begin{array}{r}\phi \text { (seguido de } \\
\text { vogal anterior), } \\
\phi^{\mathrm{w}} \text { (seguido de } \\
\text { vogal posterior) } \\
{ }^{n} b\end{array}$} & \multirow[t]{2}{*}{ 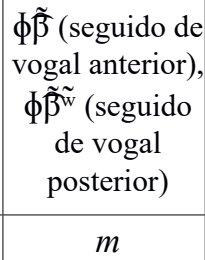 } & \multicolumn{2}{|c|}{$\begin{array}{c}\phi \sim \beta \sim \mathrm{f} \sim \mathrm{v} \sim \mathrm{v} \text { (seguido } \\
\text { de vogal anterior), } \phi \sim \beta \sim \\
\mathrm{f} \sim \mathrm{v} \sim \mathrm{v} \sim \phi^{\mathrm{w}} \sim \beta^{\mathrm{w}} \sim \mathrm{f}^{\mathrm{w}} \sim \\
\mathrm{V}^{\mathrm{w}} \sim \mathrm{v}^{\mathrm{w}} \text { (seguido de vogal } \\
\text { posterior) }\end{array}$} & \multicolumn{2}{|c|}{$\begin{array}{c}ð, \theta \text { (depois de } \\
\text { segmento fonético } \\
\text { surdo) }\end{array}$} \\
\hline$*_{n} b$ & $*_{m}$ & & & ${ }^{n} b \sim m$ & $m$ & ${ }^{n} b$ & $m$ \\
\hline$*_{n} d$ & $*_{n}$ & ${ }^{n} d$ & $n$ & ${ }^{n} d \sim n$ & $n$ & ${ }^{n} d$ & $n$ \\
\hline$*_{y}$ & $*_{n}$ & ${ }^{n} f$ & $\mathrm{n}$ & ${ }^{n} \widehat{d} \sim n$ & $\mathrm{n}$ & ${ }^{n} f$ & $\mathrm{n}$ \\
\hline$*_{n} g$ & $* \eta$ & ${ }^{n} g$ & $\mathrm{y}$ & ${ }^{n} g \sim \eta$ & $\mathrm{y}$ & ${ }^{n} g$ & $\mathrm{y}$ \\
\hline$*_{w}$ & $*_{\tilde{w}}$ & $w \sim \beta$ & $\tilde{w} \sim \tilde{\beta}$ & $w$ & $\tilde{w}$ & $v \sim v$ & $\tilde{v} \sim \mathrm{m}$ \\
\hline$*_{r-}$ & $* \tilde{r}-$ & ${ }^{\partial} r-$ & $\tilde{\partial} \tilde{\Gamma}-\sim \tilde{\partial} \tilde{\lambda_{-}}$ & f- $\sim 1-$ & 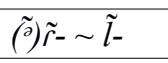 & $\underset{n}{l}$ & $\tilde{l}$ \\
\hline$*_{-r-}$ & $*_{-} \tilde{r}_{-}$ & $-\kappa-$ & $-\tilde{\Gamma}-\sim-\tilde{d}-$ & $-\Gamma-$ & $-\tilde{\tilde{r}}-$ & $\begin{array}{c}l, t \\
\text { (depois de } \\
\text { velares) }\end{array}$ & $\begin{array}{c}\tilde{l}, t \\
\text { (depois de } \\
\text { velares) }\end{array}$ \\
\hline$*_{j}$ & $* \tilde{j}$ & $j$ & $\tilde{J} \sim n$ & $j$ & $\tilde{j} \sim n$ & $j$ & $\tilde{j} \sim n$ \\
\hline$* h$ & $* \tilde{h}$ & $h$ & $\tilde{\mathrm{h}}$ & & & $h$ & $\tilde{\mathrm{h}}$ \\
\hline
\end{tabular}

Quadro 4. Correspondências entre os fones consonantais das línguas Jê Meridionais registradas para a posição de coda

\begin{tabular}{|c|c|c|c|c|c|c|c|c|c|c|c|}
\hline \multicolumn{3}{|c|}{$\begin{array}{c}\text { PJM } \\
\text { (proposta) }\end{array}$} & \multicolumn{3}{|c|}{ Kgg } & \multicolumn{3}{|c|}{$\mathrm{KgP}$} & \multicolumn{3}{|c|}{ Xok } \\
\hline $\mathrm{O}_{-}$; & $\mathrm{N}$ & $\mathrm{S}$ & $\mathrm{O}_{-}$ & $\mathrm{N}$ & $\mathrm{S}$ & $\mathrm{O}$ & $\mathrm{N}$ & $\mathrm{S}$ & $\mathrm{O}$ & $\mathrm{N}$ & $\mathrm{S}$ \\
\hline$* b^{n}$ & $*_{m}$ & $* b$ & $b^{n}$ & $m$ & $p \sim b$ & $p \sim b \sim b^{n}$ & $m$ & $p \sim b$ & $b^{n}$ & $m$ & $p \sim b \sim m$ \\
\hline$* d^{n}$ & $* n$ & $* d$ & $d^{n}$ & $n$ & $t \sim d$ & $t \sim d \sim d^{n}$ & $n$ & $t \sim d$ & $d_{n}^{n}$ & $n$ & $\underset{n}{t} \sim \underset{n}{d} \sim \underline{n}$ \\
\hline$*^{n}{ }^{n}$ & $* n$ & $*_{f}$ & $j^{n}$ & $n$ & $\begin{array}{c}{ }^{j} \mathcal{C} \sim j_{t} \tau \sim \\
j_{\mathcal{7}} \sim j^{j} d \bar{\zeta}\end{array}$ & $\begin{array}{l}{ }^{j} t \tau \sim{ }^{j} d \widehat{\zeta} \sim \\
{ }^{j} d \widehat{\zeta}^{n} \sim{ }^{i} t \sim \\
{ }^{j} d \approx{ }^{j} d^{n} \sim j\end{array}$ & $n$ & $\begin{array}{c}{ }^{j} t \tau \sim \\
{ }^{j} d \widetilde{\zeta} \sim j^{j} t \\
\sim j d \sim j\end{array}$ & $\begin{array}{l}j_{y^{n}} \sim j \\
\sim^{j} d \bar{\zeta}^{n}\end{array}$ & $n$ & $\begin{array}{c}{ }^{j} y^{n} \sim j \sim \\
{ }_{j} d \mathcal{\zeta}^{n}\end{array}$ \\
\hline$*^{*}{ }^{n}$ & $* \eta$ & $* g$ & $g^{n}$ & $\eta$ & $k \sim g$ & $k \sim g \sim g^{n}$ & $\eta$ & $k \sim g$ & $g^{n}$ & $\eta$ & $k \sim g$ \\
\hline$*_{w^{2}}$ & $* \tilde{w}^{\tilde{\partial}}$ & - & $w^{2} \sim \beta^{\circ}$ & $\tilde{w^{z}} \sim \tilde{\beta} \tilde{\partial}$ & - & $w^{2}$ & $\tilde{w^{z}}$ & - & $\tilde{v^{2}} \sim v^{\tilde{\partial}}$ & $\tilde{v^{\partial}}$ & - \\
\hline$* r^{2}$ & $* \tilde{r^{a}}$ & - & $r^{2}$ & $\tilde{\Gamma^{\partial}} \sim \tilde{d^{\partial}}$ & - & $r^{2}$ & $\tilde{r^{z}}$ & - & $\tilde{l}$ & $\tilde{l}^{2}$ & - \\
\hline$* j^{2}$ & $* \tilde{j}$ & - & $j^{2}$ & $\tilde{J^{\partial}} \sim n^{\tilde{\partial}}$ & - & $j^{\curvearrowright}$ & $\tilde{j^{\partial}} \sim n^{\tilde{\partial}}$ & - & $j^{2}$ & $\tilde{j^{\partial}} \sim n^{\tilde{\partial}}$ & - \\
\hline
\end{tabular}

Daqui em diante citaremos as formas das línguas registradas assim como as formas reconstruídas para o PJM usando as transcrições fonéticas e não fonêmicas 9 .

\footnotetext{
${ }^{8}$ Reconstruímos uma consoante lateral para esta correspondência por causa do reflexo lateral (l) na língua Ingain (Jolkesky 2010:194). Este fonema corresponde a uma fricativa palatal surda em Proto-Jê Amazônico e indiscutivelmente reflete um fone palatal do Proto-Jê, já que a fricativa palatal surda alternava com *j (Nikulin 2015:7).

${ }^{9}$ As diferenças entre a nossa transcrição das vogais das línguas registradas e a de Jolkesky são as seguintes: Kaingáng $\tilde{a}$ (Jolkesky) - ̃ (este trabalho), $r-\Lambda, u-\dot{i}, \tilde{\varepsilon}-\tilde{a}$; Kaingáng Paulista $\tilde{\varepsilon}-\tilde{a}, \tilde{\gamma}-\tilde{\partial}$, Xokléng $a-e, \gamma-\partial, \tilde{a}-\tilde{e}, \tilde{e}-\tilde{I}$.
} 


\section{Apofonia nas línguas Jê Meridionais}

Algumas raízes de várias classes morfológicas sofrem apofonia vocálica em Kaingáng e Xokléng em determinados contextos morfológicos e prosódicos. Em Kaingáng Paulista este fenômeno não acontece de uma maneira regular, porém certas formas fixas ainda preservam vestígios da alternância: kut̃a 'frio'

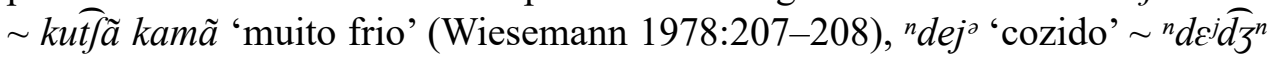

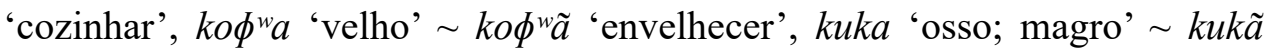
'emagrecer' (Cavalcante 1987:56). Daqui em diante utilizaremos o termo grau $A$ para nos referir a vogais inalteradas e o termo grau $B$ para nos referir a vogais modificadas.

\subsection{Kaingáng}

$\mathrm{Na}$ língua Kaingáng a apofonia atinge unicamente as raízes cuja sílaba final contenha uma vogal oral não alta $(\varepsilon, e, \supset, o, \Lambda, a)$ e não contenha uma coda pós-nasalizada (Cavalcante 1987:49-50, Jolkesky 2010:84); portanto, nem todas as raízes que correspondem a este critério são suscetíveis à apofonia. A suscetibilidade das raízes à apofonia deve ser especificada lexicalmente.

Estão apresentados, a seguir, os pares das vogais do grau A e do grau B: $e$ $>\varepsilon ; o>\jmath ; \Lambda>a ; \varepsilon>\tilde{\jmath} ; \jmath>\tilde{\jmath} ; a>\tilde{\jmath}$. A motivação fonética ou fonológica para tais mudanças não é óbvia. Segundo Rodrigues (1981:328-329), “a conversão das vogais médias pode ser descrita como resultado da aplicação de uma regra de abaixamento" e "para a conversão das vogais baixas na vogal nasalizada ã é necessário postular uma regra de nasalização”, mas, continua Rodrigues, "essa descrição é insatisfatória por duas razões: primeiro, porque recorre a duas regras independentes para dar conta de um processo morfológico único; segundo, porque a regra (2) especifica uma nasalização aparentemente imotivada". Para explicar a situação em questão, Rodrigues propõe utilizar o conceito de contínuo de ampliação da caixa de ressonância na cavidade oral. $\mathrm{Na}$ seção 5 ofereceremos uma explicação diacrônica para este fato.

Os contextos que requerem a apofonia em Kaingáng são os seguintes.

- A posição não final dentro de um sintagma nominal atributivo:

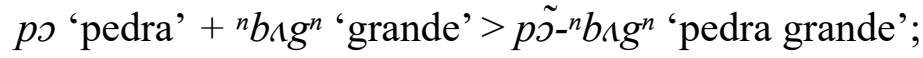

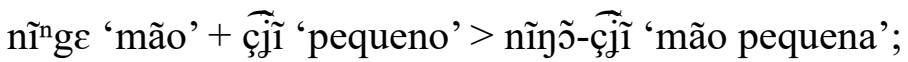

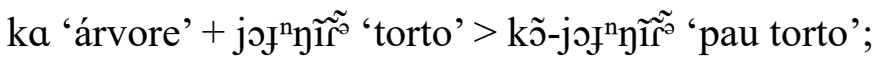

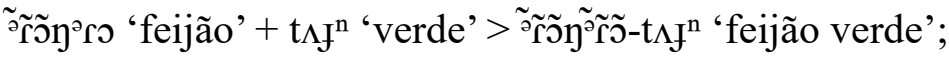

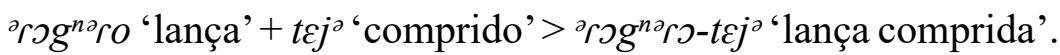

- A flexão verbal denominada $v 1$ em (Wiesemann 2011:163-164); ocorre no fim das frases entoacionais, pode ser seguida por um 
indicador de opinião, inclusive o glotal, como indicador de foco assertivo:

$\phi^{\mathrm{w}} \mathrm{a} / \phi \tilde{\tilde{\beta}^{\tilde{w}}} \tilde{\mathrm{\jmath}}(\mathrm{P})$ 'quebrar milho’;

we / we(?) 'ver';

$k o / k o(?)$ 'comer (transitivo)'.

- A posição antes de qualquer morfema cuja representação fonológica consista em uma consoante oclusiva sonora (foneticamente pósnasalizada ou nasal) que ocupe a posição de coda, incluindo as formas perfectivas de alguns verbos $\left(-g^{n} /-\eta\right)$, as formas imperfectivas de alguns verbos $\left(-d^{n} /-n,-j^{n} /-n\right)$, o sufixo ativizador (qualquer oclusiva sonora, a escolha não é previsível, excetuando-se o caso de raízes que possuem uma coda contínua; neste caso a consoante em coda é substituída por uma oclusiva sonora homorgânica10) e o clítico definitivizador $\left(-d^{n} /-n\right)$ :

$w e$ 'ver' $>w \varepsilon-g^{n}$ (forma perfectiva);

$\phi^{w} a$ 'quebrar milho' $>\phi \tilde{\beta} \tilde{w} \tilde{y}-\eta$ (forma perfectiva), $\phi \tilde{\beta} \tilde{w} \tilde{j}-n$ (forma imperfectiva);

$\phi^{w_{O}}$ 'pus' $>\phi^{w_{\supset}}-b^{n}$ 'criar pus';

nĩnge 'mão'> nĩỹ̃-n 'acenar';

jag $\phi e$ 'ninho'> jag $\phi \varepsilon-f^{n}$ 'aninhar';

$\phi^{w} a$ 'amargo'> $\phi \tilde{\bar{\beta}} \tilde{\mathrm{w}} \tilde{\partial \eta}$ 'amargar';

'row 'aberto'> ${ }^{\circ}$ ro- $b^{n}$ 'abrir';

"goj a 'água' > ngo- $f^{n}$ 'aguar';

ter 'morrer' > $\varepsilon-d^{n}$ 'matar';

${ }^{n} d \varepsilon$ 'caixa' > $n \tilde{\nu}-n$ 'a caixa';

$p$ ' 'pedra'> $p \tilde{\jmath}-n$ 'a pedra'.

\footnotetext{
${ }^{10}$ Cavalcante (1987:50-55) propõe derivar todas as variantes deste sufixo de /-n/, o que a obriga a postular formas subjacentes terminadas em fricativas surdas para algumas raízes suscetíveis à apofonia $\left(\phi^{w} O / \phi \circ \phi /\right.$ 'pus' $>\phi^{w} \supset b^{n} / \phi o \phi+\mathrm{n} /$ 'criar pus', $\phi^{w} a / \phi a h /$ 'amargo' $>\phi \tilde{\beta} \tilde{\tilde{w}} \tilde{\jmath \eta} / \phi \mathrm{ah}+\mathrm{n} /$ 'amargar') e em oclusivas surdas para algumas raízes não suscetíveis à

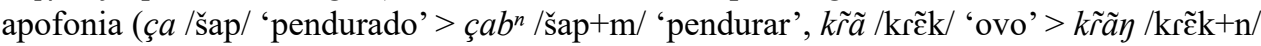
'botar ovos'). Cavalcante sugere que a diferença entre a presença e a ausência de apofonia se deva a uma diversidade entre as consoantes finais subjacentes (fricativas vs. oclusivas). No entanto, esta solução não explica a existência da mesma diferença no caso de raízes que têm uma coda contínua sonora e, portanto, não reflete o desenvolvimento diacrônico; sabese que o fonema $\phi$ do Kaingáng provém de um fonema coronal do PJM.
} 
Vale ressaltar que na língua Kaingáng as raízes que se submetem à apofonia em um destes contextos obrigatoriamente se submetem à apofonia em todos estes contextos. A seguir, alguns exemplos de raízes não suscetíveis à apofonia apesar de conterem uma vogal oral não alta na sua sílaba final: $p a$ 'sair' $>p a$ $d^{n}$ 'levar para fora'; çe 'quati' > çed $d^{n}$ 'o quati'; $\phi^{w} a$ 'perna' $>\phi^{w} a-t \varepsilon j^{\partial}$ 'perna comprida'.

\subsection{Xokléng}

Na língua Xokléng a apofonia atinge somente as raízes cuja sílaba final contenha uma vogal oral média-baixa, média ou média-alta $(\varepsilon, e, \supset, o, \partial)$ e não contenha uma coda pós-nasalizada (Jolkesky 2010:118-119); no entanto, nem todas as raízes que correspondem a esse critério são suscetíveis à apofonia. A suscetibilidade das raízes à apofonia deve ser especificada lexicalmente.

Estão apresentados, a seguir, os pares das vogais do grau A e do grau B: $e>$ $\varepsilon ; o>u ; \jmath>\supset ; \varepsilon>\boldsymbol{e} ; \supset>\boldsymbol{e}$. Assim como no caso de Kaingáng, não há motivação fonética ou fonológica evidente para tais alternâncias.

Os contextos que requerem a apofonia em Xokléng são os seguintes.

- A flexão verbal imperativa ${ }^{11}$ :

ve / $v \varepsilon$ ? 'ver';

kuce / kuce? 'beliscar.SG';

ð / ðе? 'lavar roupa'.

- A posição antes de qualquer morfema cuja representação fonológica consista em uma consoante oclusiva sonora (foneticamente pósnasalizada ou nasal) que ocupe a posição de coda, incluindo as formas perfectivas de alguns verbos $\left(-g^{n}\right)$ e o sufixo ativizador (qualquer oclusiva sonora, a escolha não é previsível, excetuandose o caso de raízes que possuem uma coda contínua; neste caso a consoante em coda é substituída por uma oclusiva sonora homorgânica):

$v e$ 'ver' $>v \varepsilon-g^{n}$ (forma perfectiva);

kuce 'beliscar.SG'> kuce-gn (forma perfectiva);

ð 'lavar roupa' $>ð \varepsilon-g^{n}$ (forma perfectiva);

ð 'amargo' >

\footnotetext{
${ }^{11}$ Segundo Jolkesky (2010:120), “as formas que em Kaingang são decorrentes de processo prosódico (em margem direita de I) parecem ter sido reinterpretadas em Xokleng como alomorfes vinculados a uma modalidade, pois nesta língua a ocorrência do verbo nesta posição prosódica tornou-se restrita e coincidente com este tipo de construção imperativa".
} 


$$
\begin{aligned}
& \text { kukle 'podre' > kukle- } \jmath^{n} \text { 'apodrecer'; }
\end{aligned}
$$

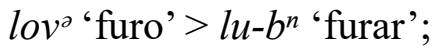

$$
\begin{aligned}
& \text { "blaj 'quebrado' > nblo-fn 'quebrar'. }
\end{aligned}
$$

A apofonia na posição não final dentro de um sintagma nominal atributivo não é mais produtiva em Xokléng, mas em certas expressões fixas mantiveramse vestígios deste processo (Wiesemann 1978:207-208).

Veja alguns exemplos de raízes não suscetíveis à apofonia apesar de conterem uma vogal oral média-baixa, média ou média-alta na sua sílaba final: kele 'descer' > keleb ${ }^{n}$ 'fazer descer'; cə 'preto' > cə- $d^{n}$ 'pretejar', $\tilde{e} k l \varepsilon$ 'pensar' $>\tilde{e} k l \varepsilon-g^{n}$ (forma perfectiva).

\section{Apofonia e vogais orais no PJM}

É fácil ver que cinco dos seis pares de vogais que participam nas alternâncias apofônicas em Kaingáng correspondem aos pares de vogais que participam no processo paralelo em Xokléng:

Kaingáng $e>\varepsilon \sim$ Xokléng $e>\varepsilon$ (na reconstrução do PJM de Jolkesky: $\left.*_{e}>*_{\varepsilon}\right)$;

Kaingáng $o>\jmath \sim$ Xokléng $o>u$ (na reconstrução do PJM de Jolkesky: $*_{o}>*^{*}$ );

Kaingáng $a>a \sim$ Xokléng $\partial>\curvearrowright$ (na reconstrução do PJM de Jolkesky: $\left.*_{\gamma}>* a\right)$;

Kaingáng $\varepsilon>\tilde{\jmath} \sim$ Xokléng $\varepsilon>\boldsymbol{e}$ (na reconstrução do PJM de Jolkesky: $\left.*_{\varepsilon}>* \tilde{a}\right)$;

Kaingáng $a>\tilde{\jmath} \sim$ Xokléng $\supset>\varepsilon$ (na reconstrução do PJM de Jolkesky: $* a>* \tilde{a})$.

Não se tem registrado uma correspondência para o par $\supset>\tilde{\jmath}$ de Kaingáng em Xokléng (esperar-se-ia $* u>*^{*} a$ ).

As reconstruções acima ilustram que, segundo Jolkesky, a apofonia incluía o abaixamento das vogais médias-altas, o abaixamento e a nasalização das vogais médias-baixas e a nasalização da vogal baixa ${ }^{12}$.

No entanto, os dados de Xokléng indicam que a distinção entre as vogais do grau A e as vogais do grau B no PJM não necessariamente envolvia o traço de nasalização. Suponhamos que a correspondência Kaingáng $\tilde{\jmath} \sim$ Kaingáng Paulista

\footnotetext{
${ }^{12}$ Note-se que na reconstrução de Jolkesky as vogais foneticamente médias-baixas se tratam como fonologicamente baixas. Deste modo, trata-se de apenas dois processos no nível fonológico: abaixamento das vogais médias e nasalização das vogais baixas.
} 
$\tilde{a} \sim$ Xokléng e provenha de um protofonema oral $(* a)$ e que a apofonia no PJM consista em um simples abaixamento vocálico. Neste caso o $* a$ na reconstrução de Jolkesky teria sido uma vogal média-baixa (*a). Apresentamos, a seguir, a proposta de reconstrução do sistema de vogais orais do PJM (incluímos as variantes livres fonéticas que se encontram na posição tônica para as línguas registradas).

Quadro 5. Vogais orais do PJM (sistema revisado)

\begin{tabular}{|c|c|c|c|c|}
\hline $\begin{array}{c}\text { PJM } \\
\text { (Jolkesky) }\end{array}$ & $\begin{array}{c}\text { PJM } \\
\text { (proposta) }\end{array}$ & Kgg & $\mathrm{KgP}$ & Xok \\
\hline$* \tilde{a}$ & $* a$ & $\tilde{\partial}(\sim \tilde{a} \sim \tilde{e})$ & $\tilde{a}(\sim \tilde{\varepsilon})$ & 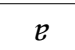 \\
\hline$* a$ & $*_{1}$ & $a(\sim e)$ & $a(\sim e)$ & כ \\
\hline$*_{\varepsilon}$ & $*_{\varepsilon}$ & $\varepsilon(\sim \alpha)$ & $\varepsilon(\sim \alpha)$ & $\varepsilon$ \\
\hline$* 0$ & $*_{0}$ & 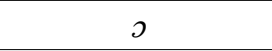 & o & $u$ \\
\hline$*_{\gamma}$ & $*_{\gamma}$ & $\Lambda(\sim 9)(i$ se átono $)$ & $\gamma(\sim e \sim \dot{i})(\dot{t}(\sim 9)$ se átono $)$ & $\partial$ \\
\hline$*_{e}$ & $*_{e}$ & $e$ & $e(\sim e)$ & $e$ \\
\hline$*_{O}$ & $*_{O}$ & $o(\sim \mho)$ & $o$ & $o$ \\
\hline$* u$ & $* u$ & $\dot{t}$ & $u(\sim i)$ & $\dot{t}$ \\
\hline$*_{i}$ & $*_{i}$ & $i$ & $i$ & $i$ \\
\hline$* u$ & $* u$ & $u$ & $u$ & $u$ \\
\hline
\end{tabular}

Os cinco pares apofônicos reconstruíveis para o PJM seriam então os seguintes: $*_{e}>*_{\varepsilon}, *_{o}>*_{j}, *_{\gamma}>*_{\Lambda}, *_{\varepsilon}>*_{a}, *_{\Lambda}>*_{a}$. O sexto par, $*_{\jmath}>*_{a}$, também deve ter existido no PJM, mas provavelmente se perdeu em Xokléng devido à mudança fonética $*_{\jmath}>u$ que fez a diferença entre os membros do par grande demais. Desse modo, a apofonia teria sido essencialmente um processo de abaixamento que afetava todas as vogais orais médias-baixas e médias-altas em raízes suscetíveis à mesma.

É interessante observar como as relações sistêmicas entre os fonemas de uma língua não deixam de existir, mesmo que as motivações fonéticas para estas relações sejam perdidas. Neste caso, a nasalização da vogal baixa oral em Kaingáng não impediu a ocorrência das alternâncias, mas fez com que o contínuo de abertura, relevante para a descrição das alternâncias no Proto-Jê Meridional, se transformasse no contínuo de ampliação da caixa de ressonância na cavidade oral, proposto por Rodrigues (1981).

Vale ainda ressaltar que, aparentemente, as condições para a apofonia no PJM eram as mesmas que ainda estão em vigor em Kaingáng (com a exceção do clítico definitivizador que deve ser uma inovação recente), a saber: a posição não final dentro de um sintagma nominal atributivo, a posição final de um verbo dentro de uma frase entoacional e a posição antes de uma coda pós-nasalizada. Esta última observação é muito importante para a reconstrução do vocalismo do ProtoJê, já que o abaixamento pode ter historicamente ocorrido não somente 
em determinados contextos morfológicos, mas também antes de qualquer coda pós-nasalizada, mesmo em palavras não derivadas.

\section{Vogais nasais no PJM}

Como, segundo a nossa proposta, a correspondência Kaingáng $\tilde{j} \sim$ Kaingáng Paulista $\tilde{a} \sim$ Xokléng $\varepsilon$ não provém de $* \tilde{a}$ e sim de $* a$ do PJM, podemos supor que * $\tilde{a}$ gerou a correspondência Kaingáng $\tilde{e} \sim$ Kaingáng Paulista $\tilde{a} \sim$ Xokléng $\tilde{\boldsymbol{B}}(* \tilde{\varepsilon}$ na reconstrução de Jolkesky).

Há ainda duas correspondências que, em nossa opinião, devem ser reinterpretadas:

$$
\begin{aligned}
& \text { Kaingáng } \tilde{\gamma} \sim \text { Kaingáng Paulista } \tilde{\partial} \sim \text { Xokléng } \tilde{\partial} \text { ( *̃̃ na reconstrução } \\
& \text { de Jolkesky); } \\
& \text { Kaingáng } \tilde{\gamma} \sim \text { Kaingáng Paulista } \tilde{\partial} \sim \text { Xokléng } \tilde{u}(* \tilde{u} \text { na reconstrução } \\
& \text { de Jolkesky). }
\end{aligned}
$$

Lembrando que as vogais orais $\supset$ e $u$ em Xokléng podem provir de $*_{\Lambda} \mathrm{e}$ *), respectivamente, e supondo que em Xokléng as vogais nasais do PJM se desenvolveram da mesma maneira que as vogais orais, parece-nos lógico

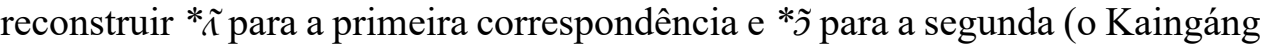
e o Kaingáng Paulista teriam perdido neste caso o traço arredondado).

Preferimos também reconstruir uma vogal nasal quase-alta $*_{I}$ para a correspondência Kaingáng $\tilde{\imath} \sim$ Kaingáng Paulista $\tilde{\imath} \sim$ Xokléng $\tilde{\varepsilon}$, já que uma variante livre quase-alta é registrada tanto em Xokléng quanto em Kaingáng.

Abaixo apresentamos o quadro do sistema de vogais nasais do PJM

\begin{tabular}{|c|c|c|c|c|}
\hline PJM (Jolkesky) & PJM (proposta) & $\mathrm{Kgg}$ & $\mathrm{KgP}$ & Xok \\
\hline$* \tilde{j}$ & $* \tilde{\imath}$ & $\tilde{\gamma}(\sim \tilde{\mu})$ & $\tilde{\partial}(\sim \tilde{e})$ & $\tilde{\partial}(\sim \tilde{o} \sim \tilde{\partial})$ \\
\hline$* \tilde{\varepsilon}$ & $* \tilde{a}$ & $\tilde{a}(\sim \tilde{\varepsilon})$ & $\tilde{a}(\sim \tilde{\varepsilon})$ & $\tilde{\varepsilon}(\sim \tilde{\partial})$ \\
\hline$* \tilde{u}$ & $* \tilde{j}$ & $\tilde{\gamma}(\sim \tilde{\mu})$ & $\tilde{\partial}(\sim \tilde{e})$ & $\tilde{u}$ \\
\hline$* \tilde{l}$ & $*_{I}^{\tilde{I}}$ & $\tilde{l}(\sim \tilde{I})$ & $\tilde{l}$ & $\tilde{\varepsilon}(\sim \tilde{I})$ \\
\hline$* \tilde{u}$ & $* \tilde{u}$ & $\tilde{u}(\sim \tilde{\sigma})$ & $\tilde{u}(\sim \tilde{o} \sim \tilde{\jmath})$ & $\tilde{u}$ \\
\hline
\end{tabular}
(incluímos as variantes livres fonéticas que se encontram na posição tônica para as línguas registradas).

Quadro 6. Vogais nasais do PJM (sistema revisado)

\section{Correspondências externas}

Estão apresentadas abaixo as correspondências regulares estabelecidas entre as vogais do PJM e as do Proto-Jê Amazônico (PJA) (Nikulin 2015) ${ }^{13}$.

${ }^{13}$ Citamos abaixo os dados da língua Ingain apesar de pensarmos que a última língua ancestral comum de Xokléng e Kaingáng, tratada acima, não é uma língua ancestral direta de Ingain (Jolkesky 2010:269). Usamos as seguintes abreviações para as fontes usadas: AM1 
Apresentamos ainda, pela primeira vez, desde o trabalho pioneiro de Davis (1966), uma proposta de reconstrução de vogais do Proto-Jê (PJ), acrescentando as correspondências na língua Maxakalí e no Proto-Jabutí (PJab), usando uma versão modificada por mim (Nikulin 2005:37-38) ${ }^{14}$ da reconstrução proposta por Voort (2007).

7.1.PJ * $a$ teria se nasalizado invariavelmente para $* \tilde{a}$ em PJM, permanecendo intacto em PJA. A nasalização em questão teria ocorrido na protolíngua de Kaingáng e Xokléng depois da separação de Ingain (observe as palavras para 'asa', 'filho', 'ter medo', entre outras), apesar de ser incerto se as vogais nasais existiam nesta língua.

Corresponde a $a$ na língua Maxakalí e no PJab:

PJ *par 'pé' Maxakalí pata (e PJab *praj com síncope);

PJ *çare / *jare 'raiz' Maxakalí mĩm= $\tilde{\imath} p=$ fatit $\sim$ PJab *ari / $*=\bar{d}$ ari

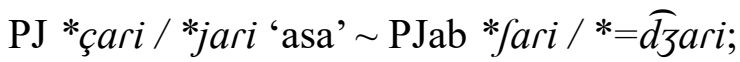

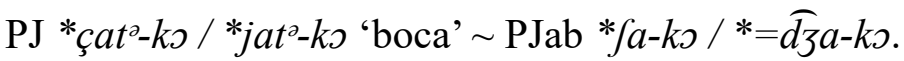

Em alguns casos, no entanto, corresponde a $o$ em Maxakalí e a *jj em PJab:

PJ *kra 'filho, descendente' Maxakalí kitok $\sim$ PJab *krəj;

$\mathrm{PJ}$ *çĩp $=k r a /$ nĩ $=k r a$ 'mão' Maxakalí jĩp=kitok 'dedo'.

Nos seguintes casos aparentemente foi nasalizado em Maxakalí:

PJ *pa 'braço, galho' $\sim$ Maxakalí =mãy $\sim$ PJab * $f a=p a /$ $*=\widehat{d z} a=p a$;

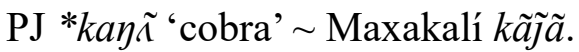

Nos seguintes casos omônimos ocorreu um alteamento no Proto-Jabutí:

$\mathrm{PJ}{ }^{* n} b a$ 'ouvir, escutar' $\sim$ Maxakalí $/ \dot{t}=p a k \sim$ PJab ${ }^{* n} b ə ;$

PJ *n $b a$ 'fígado' $\sim$ PJab *n bo.

Vejam-se alguns exemplos da correspondência PJM * $\tilde{a} \sim \mathrm{PJA} * a$ :

(Ambrosetti 1896, dados de Pedro), AM2 (Ambrosetti 1896, dados de María Antonia), AM3 (Ambrosetti 1896, dados de Cosme Román), AM4 (Ambrosetti 1896, dados de Luchessi), VO1 e VO3 (Vogt 1904), LI (Lista 1883). A representação fonética é altamente preliminar. ${ }^{14}$ Preferimos substituir o símbolo $* \check{r}$, proposto por nós para a correspondência Arikapú $-\widehat{t f}$-, $-\varnothing-\sim$ Djeoromitxí $-r$ - por $* \widehat{d z}$. A mudança fonética $\widehat{d \zeta}>r$ ocorreu na história de línguas como o húngaro (Aikio 2015, no prelo) ou o yukaghir de tundra (Nikolaeva 2006), apesar de a mudança na direção oposta também ter sido registrada na língua udmurt (Bubrix 1948:28). 
$\mathrm{PJM} * t \tilde{a} \tilde{r^{\partial}}$ 'asa' $\left(\operatorname{Kgg} \tilde{\phi} \tilde{c} \tilde{r^{\partial}}\right.$ 'asa, pena', $\operatorname{KgP} \phi^{w} \tilde{a} \tilde{r}^{\partial} \sim \tilde{w} \tilde{a} \tilde{r}^{\tilde{r}}$, Xok $\partial \tilde{e} \tilde{l}^{\text {, }}$, Ing lar $\left.{ }^{215}\right) \sim \mathrm{PJA} *$ *̧ari $/ *_{\text {jari }}$;

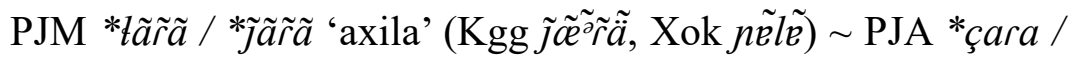
*jara;

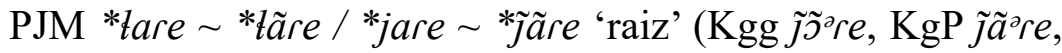
Xok jele $\sim$ jẽle, ðele-dn 'enraizar') $\sim \mathrm{PJA}$ *çare / *jare (compare também $\mathrm{KgP} \tilde{j} \tilde{a} \tilde{r} \tilde{r} \tilde{e}$ e Tapayúna tarĩ com o mesmo significado; aparentemente, uma variante *çarĩ / *jarĩ existia já no ProtoJê);

$\mathrm{PJM} * t \tilde{a} / * \tilde{\jmath} \tilde{a}$ 'em pé (singular)’ $(\operatorname{Kgg} \tilde{\phi} \tilde{c} / \tilde{j} \tilde{c} \tilde{e}, \operatorname{KgP} \tilde{\jmath} \tilde{a}, \mathrm{Xok} n \tilde{\varepsilon}$, $n \tilde{\varepsilon}-\eta / \partial \tilde{e}-\eta$ 'colocar de pé') $\sim$ PJA *ça / *ła;

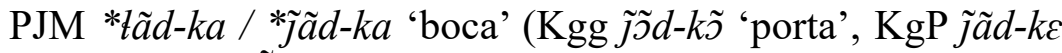
'porta', Xok $\partial \tilde{e} d-k e$ 'porta', Ing jet-kau'16 'boca') PJA *çatº $k w a / * j a t^{\curvearrowright}-k w a$;

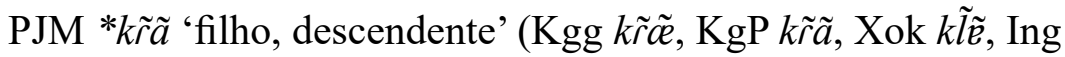
$\left.k r a^{17}\right) \sim$ PJA *kra;

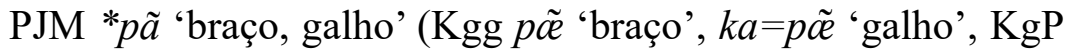
$j \jmath=p \tilde{a}$ 'mão', Xok $p \tilde{e}$ 'braço', Ing `'ba's 'braço, ombro') $\sim$ PJA *pa;

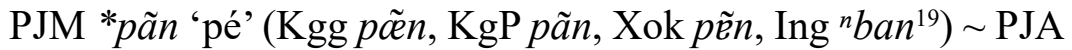
*par*;

PJM *mã 'ouvir' $\left(\operatorname{Kgg} m \tilde{e}, \operatorname{KgP} m \tilde{a}\right.$, Xok $\left.m \tilde{e}, \operatorname{Ing}=^{n} b a^{20}\right) \sim$ PJA *n $b a$ 'ouvir, escutar, entender';

PJM *t $t=m \tilde{a}$ 'fígado' $(\operatorname{Kgg} t \tilde{\gamma}=m \tilde{e}, \operatorname{KgP} t a=m \tilde{a}$, Xok $t \tilde{\jmath}=m \tilde{\varepsilon}$, Ing $\left.t \tilde{a}=^{n} b^{2} n^{21}\right) \sim$ PJA ${ }^{* n} b a$ 'ouvir, escutar, entender';

PJM *mã 'ter medo' (Kgg $k a=m \tilde{e}-\eta, \operatorname{KgP} m \tilde{a}-n$, Xok $m \tilde{e}-\eta$, Ing $\left.k u={ }^{n} b a^{22}\right) \sim$ PJA ${ }^{* n} b a$.

\footnotetext{
${ }^{15} \mathrm{AM} 2$ làrá, VO1 làr.

${ }^{16}$ AM1 am=it-au, AM2 niet-kau, AM4 (?) am=ign-ú, VO1 net-kó, VO3 yit-kaū, LI am=incá. Considere também AM2 yet-kan 'porta'.

${ }^{17} \mathrm{AM} 1 i=k r a ́, \mathrm{VO} 1$ krá, LI an=trá.

${ }^{18}$ AM2, LI am=má, VO1, VO3 bá 'ombro'. Considere também AM2 mbó 'ombro'.

${ }^{19} \mathrm{AM} 1 a=m b a ́ n, \mathrm{AM} 2 a=m b a r, \mathrm{VO} 1$ bàn, VO3 bján, buàn.

${ }^{20}$ AM2 duambanjamó 'escutar'.

${ }^{21} \mathrm{AM} 2$ ta=mpan.

${ }^{22}$ AM2 ku=mbá 'ter medo, medroso'.
} 
Encontramos três casos que apresentam a correspondência PJM * $a \sim$ PJA * $a$, mas todos eles possuem mais alguma irregularidade.

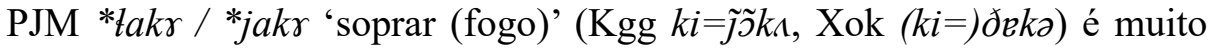
semelhante a PJA *çako 'soprar', mas a correspondência PJM $*_{\gamma} \sim$ PJA *o não é encontrada em nenhuma outra palavra. Embora a palavra em questão seja suscetível à metafonia em Kaingáng (e dessa maneira talvez possuísse os alomorfes *lakn- / *jakn- no PMJ), em todos os outros casos são as vogais do grau A e não do grau B que refletem as vogais do PJ. Compare Djeoromitxí $\hbar \varepsilon k u$ 'soprar' (talvez $<$ PJab * ${ }^{t} \varepsilon k u$ ).

PJM *n grad $^{n}$ 'arara' (Xok teg=ngled , Ing $\mathrm{kla}^{23}$ ) poderia ser comparado com *krata / *krada 'arara' do Proto-Jê Central (PAJ *ngrat ou *krat ${ }^{2}$ ), mas a correspondência em coda seria sem precedente. Além disso, a segmentação da palavra Xokléng proposta por Jolkesky (2010:222) nos parece ad hoc e o parentesco entre as palavras Xokléng e Ingain não é óbvio. Já a comparação entre as palavras Ingain, Xavánte e Xerénte sem a inclusão da palavra Xokléng não apresenta nenhuma dificuldade fonológica.

PJM *kıka 'rosto, testa' (Kgg kakõ, KgP kuka kakã, Xok koke, Ing $k u k a^{24}$ ) é semelhante a Proto-Jê Norocidental *kuka 'testa' (que pode provir de uma forma como PJA *kuka), mas a correspondência das vogais na primeira sílaba é irregular. Talvez só a palavra Ingain tenha que ser incluída na comparação.

7.2. PJ *3 teria gerado PJM * $a$ em todos os contextos. No PJA o reflexo é *wa em sílabas abertas, *wr em sílabas fechadas e *u seguindo uma consoante labial. Note-se que os núcleos $* w a$ e *wr não combinam com onsets labiais no PJA; esta restrição ainda existe nas línguas modernas, tais como Pykobjê (Sá, 1999:14).

$\mathrm{PJ}$ *J corresponde regularmente a $o$ na língua Maxakalí e a $\supset$ em PJab:

PJ *kor 'mandioca' Maxakalí kot / kohot;

PJ *ts $b^{n a}$ 'gordura' $\sim$ Maxakalí top $\sim$ PJab *tõ $\sim$ *to- (?);

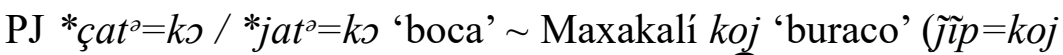
'orelha', $\tilde{j} \tilde{l} \tilde{j}=k o j$ 'boca') $\sim$ PJab * $a-k s / *=\bar{d} z a-k s$;

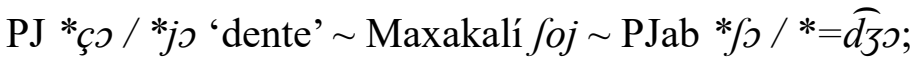

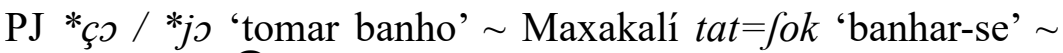
$\mathrm{PJab} * \int \frac{\pi}{*}=\widehat{d} 30$;

PJ *kojks 'céu' Maxakalí pejkoj (compare Djeoromitxí bek $=k \varnothing$, talvez $<\mathrm{PJab} *=k s$ ).

\footnotetext{
${ }^{23}$ AM1 kluá, AM2 kián (?), VO1 kakladêin, VO3 klá.

${ }^{24}$ AM2 $a=k u k a$ 'testa', VO1 kokó 'rosto', VO3 tokuá 'testa' (?), LI $a=c u c a ́ ~ \sim a=p u c a ́ '$ 'testa'.
} 
Vejam-se alguns exemplos:

PJM *a PJA *wa (em sílabas abertas seguindo uma consoante não labial):

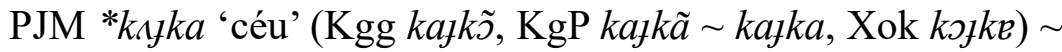
PJA *kVlkwa;

PJM *ja 'dente' $\left(\operatorname{Kgg} \tilde{j} \tilde{,}, \mathrm{KgP} \tilde{j} \tilde{a}, \mathrm{Xok} j e\right.$, Ing jau $\left.{ }^{25}\right) \sim \mathrm{PJA} * c ̧ w a$ / *fwa;

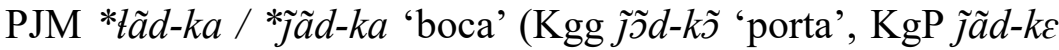
'porta', Xok $\partial \tilde{e} d-k e$ 'porta', Ing jet-kau 'boca'26) PJA * ${ }^{2} a t^{2}$ $k w a / * j a t^{2}-k w a$;

PJM *kıja 'azedo, salgado' (Kgg kaj̃̃ 'azedo, salgado', KgP kajã $-N$ 'azedar') PAJ *kajwa 'sal'.

PJM * $a \sim$ PJA *wr (em sílabas fechadas, único exemplo):

PJM * $\operatorname{tag}^{n}$ 'gordura, gordo' (Kgg tõ $\eta$ 'gordo', KgP tã $\eta$ 'gordo', Xok $t e g^{n}$, Ing $\left.{ }^{n} d a n^{27}\right) \sim$ PAJ *twr $b^{n a}$ 'gordura'.

PJM * $a \sim$ PJA $* u$ (seguindo uma consoante labial):

PJM *patu 'mutuca' (Kgg p̃tu, Xok petu, Ing pedu ${ }^{28}$ ) PAJ * putu 'maruim, pium';

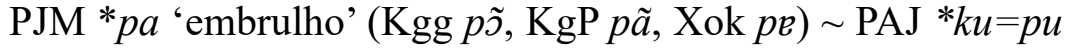
'embrulhar'.

7.3. PJ $*_{o}$ teria gerado PJM $*_{\Lambda}$ em todos os contextos, permanecendo do PJA.

PJ $* o$ corresponde regularmente a $\dot{t}$ na língua Maxakalí; em PJab encontramos *u:

PJ *kop 'mosca, borrachudo' Maxakalí kỉb-jãm 'mosca';

PJ *pok 'acender' Maxakalí pik 'queimar, cozinhar';

PJ *çoj 'folha' Maxakalí jijj;

PJ *ço 'chupar' Maxakalí fip $\sim$ PJab *?u;

\footnotetext{
${ }^{25}$ AM1 am=iau, AM2 amn=iau, VO1 ńó, VO3 ńáu, yau, LI ami=yao.

${ }^{26}$ AM1 am=it-au, AM2 niet-kau, AM4 (?) am=ign-ú, VO1 net-kó, VO3 yit-kau, LI am=incá. Considere também AM2 yet-kan 'porta'.

${ }^{27}$ AM2 iun=gue=dá 'óleo de tambu', VO1 dàn 'gordo'; ndàn, ndan 'gordura'; jun=gě=dàn 'gordura do tambu', VO3 ndjàn 'gordura'; jyn=gy=dá 'gordura do tambu'.

${ }^{28}$ AM2 pedú 'mosquito', se não é cognado de Xavánte e Xerénte pidu 'mutuca'.
} 


$$
\begin{aligned}
& \text { PJ *ko 'árvore' } \sim \text { Maxakalí kip } \sim \text { PJab *ku; } \\
& \text { PJ *ngo 'piolho' Maxakalí kit; } \\
& \text { PJ *nbok 'lago' } \sim \text { Maxakalí pij-hep. }
\end{aligned}
$$

Vejam-se alguns exemplos da correspondência PJM $*_{\Lambda} \sim \operatorname{PJA} *_{o}$ :

PJM *ka=k৯ 'vento' (Kgg $k \tilde{\jmath} k a$; Xok $k \tilde{e} k e^{29}$, Ing $\left.k a k a^{30}\right) \sim$ PJA $*(k a=) k o k^{2}$;

$\mathrm{PJM} * k \wedge$ 'árvore' (Kgg, KgP ka, Xok ko), PJM *nI $=k \wedge$ 'chifre' (Kgg, KgP $n \tilde{\imath}-k a$, Xok $n \tilde{I}=k s) \sim$ PJA *ko 'pé de arvore, chifre, borduna';

PJM * $k a$ 'mosquito, borrachudo' $(\operatorname{Kgg} k a$, Xok $c \tilde{I}=t o=k s) \sim$ PJA *kop ' 'mosca';

PJM * $=t_{\Omega}$ 'mamar' (Kgg pag $=\phi^{w} a$, pl. pig $=\phi^{w} a, \mathrm{KgP} p a=\phi^{w} a$, Xok $\left.p \varepsilon\left(g^{n}\right)=\partial \supset\right) \sim$ PJA *ço 'chupar';

PJM *ng^ 'piolho, caruncho' (Kgg 'na 'piolho, caruncho, coró', $\mathrm{KgP}{ }^{n} g a$, Xok ${ }^{n} g$, Ing $\left.{ }^{n} g a^{31}\right) \sim \mathrm{PJA}{ }^{* n} g o$ 'piolho'.

7.4. PJ $* u$ teria se conservado no PJA, convertendo-se em $*_{o}$ no PJM. Nos poucos casos conhecidos $\mathrm{PJ} * u$ corresponde regularmente a $a$ na língua Maxakalí e a $o$ no PJab:

PJ *tu 'carregar' Maxakalí taha / tat (talvez PJab *to 'levar', $* k a=t \partial$ 'carregar');

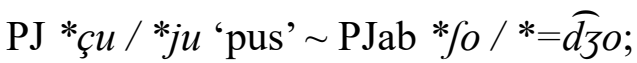

$\mathrm{PJ} * k u$ 'comer' $\sim \mathrm{PJab} * k o$;

PJ *tugra 'seco' Maxakalí dak.

Vejam-se alguns exemplos:

$\mathrm{PJM} * t_{o} / *^{*}=$ jo 'pus' $\left(\mathrm{Kgg} \phi^{w}\right.$, jãnn=jo 'látex, resina', $\mathrm{KgP}$ $\tilde{j} \tilde{a}=n \tilde{u}$ 'látex, resina', Xok $o$ ) $\sim$ PJA *çu / *ju;

PJM *ko 'comer' (Kgg, KgP, Xok ko, Ing $\left.k u^{32}\right) \sim$ PJA *ku.

\footnotetext{
${ }^{29}$ A forma Xokléng parece ser um empréstimo Kaingáng.

${ }^{30}$ VO1 kaká 'vento, trovão'.

${ }^{31}$ AM2 nga 'piolho', VO1 ngá 'piolho', VO3 ngá nganá 'piolho'.

${ }^{32} \mathrm{VO} 1$ ku-tum-bĕ 'ainda não comi'; ku-jýma 'já comi'.
} 
No seguinte caso o abaixamento não ocorreu em PJM *tu 'carregar nas costas' (Kgg $t u$, Xok $t u$, Ing $\left.{ }^{n} d u^{33}\right)$. A escassez dos exemplos conhecidos nos impossibilita de identificar o condicionamento para isso. Considere também o seguinte exemplo onde a vogal foi abaixada por causa da apofonia: PJ *tug ${ }^{\text {na }}$ $>$ PJM *tog ' 'seco (de plantas)' (Kgg, KgP $t o g^{n}$, Xok $\left.t u g^{n}\right)$. Não foi encontrada nenhuma correspondência nas línguas Jê Amazônicas, mas a vogal do cognato Maxakalí ( $d a k)$ pode corresponder somente a uma das seguintes vogais do PJ: $* a, * u$, ${ }^{*} u$, das quais somente a segunda pode ter gerado a vogal do PJM.

7.5. PJ * $*_{1}$ teria se labializado no PJA, enquanto no PJM teria se coincidido com o *a do PJ nasalizando-se para * $\tilde{a}$. Como no caso de PJ *a, a nasalização em questão deve ter ocorrido na protolíngua de Kaingáng e Xokléng depois da separação de Ingain.

$\mathrm{O} *_{\wedge}$ do PJ corresponde a $o$ em Maxakalí e a $*_{\partial}$ ou $*_{o}$ em PJab:

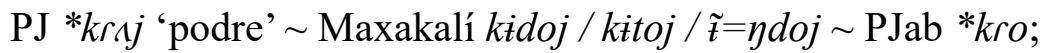

PJ *n brs 'cinza' Maxakalí pitohok / pitok PJab *nbrə;

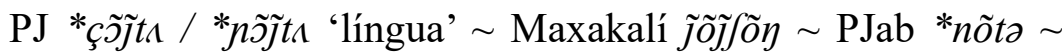
${ }^{*}$ dutz;

PJ ${ }^{* n} b_{\wedge} k^{2} \sim{ }^{*} p \wedge k^{2}$ 'flecha' Maxakalí pohoj $\sim$ PJab ${ }^{* n} b o$.

Vejam-se alguns exemplos:

PJM $* k_{\Lambda}=n \tilde{a}$ 'olho, fruta' $\left(\operatorname{Kgg} k a=n \tilde{e}, \operatorname{KgP} k a=n \tilde{a} \sim k a=d^{n} d \varepsilon\right.$, Xok $k s=n \tilde{e}$, Ing $\left.(k e=)^{n} d a^{34}\right) \sim$ PJA *n $d s b^{n}$ 'olho';

$\mathrm{PJM} * m \tilde{r} \tilde{a}$ 'cinza' $\left(\operatorname{Kgg} m \tilde{r} \tilde{c} \tilde{j} \tilde{j}, \operatorname{KgP} m \tilde{r} \tilde{a} \tilde{j} \tilde{j}\right.$, Xok $\left.m \tilde{l} \tilde{\varepsilon}, \operatorname{Ing} m r a(u)^{35}\right)$ $\sim$ PJA *n $b r \rho$;

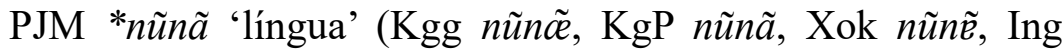

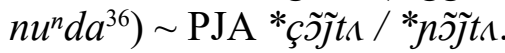

\footnotetext{
${ }^{33}$ AM2 dú 'encarregar'.

${ }^{34}$ AM2 kenda-djó 'olhos'; da-iá 'pupila'; ndá=ioró 'pálpebra'; kendá 'cara'; re=ntá 'fruto de caraguatá'; chau=ndá 'mamão'; $k u=n d a ́$ 'laranja'; ndá-bá 'espiga'; kundan-gá 'besouro', AM3 krigõ=ndá 'guabiroba', AM4 gundón 'olhos' (?), VO1 kendá 'olho'; ro=kendá 'sol'; djun=kandá 'coco'; grein=kantá 'fruto do caraguatá'; $k u c ̌ a u=k a n d a ́$ 'jaracatiá'; $k y=k a n d a ́$ 'laranja'; kunda-ngá 'caruncho'; kenda-ngáin 'cílias', VO3 kandá 'olho, rosto'; rêin-dá, rein-dá 'caraguatá, fruto do caraguatá'; krego=ndá 'guabiroba'; čau=ndá 'jaracatiá'; $k y=n d a ́$ 'laranja'; kandêa-ki, kandea-ki 'sobrancelhas', LI apintá 'olyos', apita-mingay 'pestana'.

${ }^{35}$ AM2 maráu, VO1 mrá, VO3, LI nmará.

${ }^{36} \mathrm{AM} 1$ agn=upá (?), AM2 am=undá, VO1 nomdá, VO3 naurá (?).
} 
A irregularidade no seguinte par de cognatos provavelmente deve-se à monotongação de um ditongo Proto-Jê (conservado somente em Xerénte): PJM $* k \supset=k r \varepsilon$ 'podre' $($ Kgg $k \supset=k r \varepsilon$, Xok $k u=k l \varepsilon) \sim \mathrm{PJ} * k r \jmath j$.

7.6. PJ $*_{\gamma}$ teria se abaixado para * $*_{\Lambda}$ no PJA, mantendo-se em PJM.

$\mathrm{O} * \gamma$ do PJ corresponde a $\dot{t}$ em Maxakalí e a *z em PJab:

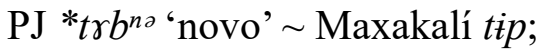

$\mathrm{PJ} * c ̧ \gamma(j) \sim *_{j \gamma}(j)$ 'urina' Maxakalí $/ \mathrm{j} j$ 'urinar';

provavelmente PJ *prr(C) 'pena' Maxakalí pitity-nãy 'pássaro' (pidiynãy na língua dos cantos (Campos 2009:31); compare Pykobjê pra-re 'pássaro');

PJ *krrtəo 'mariposa' (somente Pykobjê $k^{h} r \partial t$-jara-re) Maxakalí kittit-tap;

PJ *tr 'mulher' Maxakalí tit 'mãe, irmã da mãe, animal de grande porte';

PJ *kr 'pele, corpo' PJab *kə 'pele, couro, casca'.

Vejam-se alguns exemplos:

PJM *jr $(j)$ 'urina' $\left(\operatorname{Kgg} j \wedge, \operatorname{KgP} j \gamma j^{2}\right.$, Xok jof $\left.{ }^{n}\right) \sim \operatorname{PJA} * c ̧ \wedge$;

PJM *hr (Kgg $h \wedge, \operatorname{KgP} h \gamma)$ 'corpo, tronco' $\sim$ PJA *kı 'pele, corpo, peito';

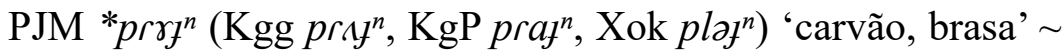
PJA *prs;

No seguinte caso a correspondência é irregular (a vogal parece ainda ter sido afetada pela metafonia):

PJM *tagn $\left(\operatorname{Kgg} t \tilde{\jmath} \eta, \mathrm{KgP} t \tilde{a} \eta\right.$, Xok teg $\left.{ }^{n}\right) \sim \mathrm{PJA} * t \_b^{n \curvearrowright}$ 'novo, cru'.

7.7. PJ $* u$ teria se conservado nas protolínguas dos dois ramos, já a correspondência desta vogal tanto em Maxakalí como em PJab é uma vogal baixa, *a em Maxakalí e *a ou *ã e PJab:

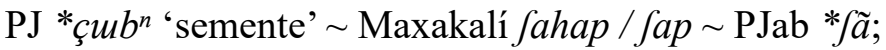

PJ *ku=çubn 'fogo' Maxakalí kîfap;

PJ *pru 'caminho' Maxakalí pitahat;

PJ *bu 'pegar' $\sim$ Maxakalí paha / pa PJab *wa; 
Maxakalí toj 'comprido' poderia ser comparado tanto com PJA *ru, como com PJM *tcj com o mesmo significado; em ambos os casos a correspondência vocálica seria irregular.

Vejam-se alguns exemplos:

PJM * $k u=t w$ 'escuro' (Kgg, Xok $k u=t i, \operatorname{KgP} k u=t w$, Ing $k u=n a^{37}$ ) $\sim$ PJA *tuk 'preto';

PJM *=pru 'caminho carreiro' (Kgg $\tilde{P} \tilde{c}=p r i \quad / j a=p r \dot{t}, \mathrm{Kgg}$ ja=pru) PJA *pru 'caminho';

PJM *n $b u$ 'rabo' (Kgg, Xog ${ }^{n} b \dot{t}, \mathrm{KgP}^{n} b u$, Ing $\left.b w^{38}\right) \sim \mathrm{PJA}{ }^{* n} b u d^{n}$;

PJM *tu 'semente' (Kgg $\phi^{w} \dot{t}, \mathrm{KgP} \phi^{w} u, n \tilde{a} \phi^{w} \gamma(?)$, Xok $\partial \dot{t}$, Ing $\left.l u(n)^{39}\right) \sim \mathrm{PJA} * c ̧ u b^{n}$;

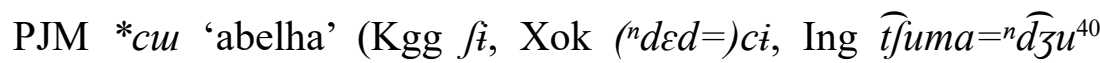
'abelha-mirim') $\sim$ PJA * $f m$;

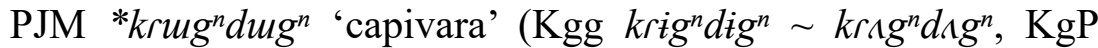
krug gru (?), Xok kligndign 'paca') PJA *kob ${ }^{n} t u b^{n}$.

7.8. PJ $*_{\varepsilon}$ teria se conservado nos dois ramos. A correspondência regular em Maxakalí e em PJab parece ser idêntica:

PJ *ngre 'ovo, genital' PJab *ngre 'ovo';

PJ *ngre 'dançar, cantar' Maxakalí kitej 'cantar, tocar, entoar os rituais' PJab *ngre 'dançar';

PJ *n beni 'mel' $\sim$ PJab *n $b \varepsilon$ 'abelha, caba'.

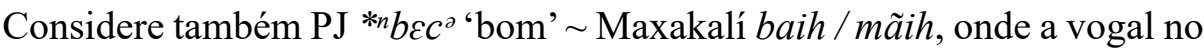
Proto-Jê pode resultar da monotongação de um ditongo antigo. Se Xavánte pire e Xerénte pre 'pesado, árduo, autoritário' realmente provêm de PJ *pirc, podese comparar esta raiz com Maxakalí pitij 'pesado'.

Vejam-se alguns exemplos:

$\mathrm{PJM}{ }^{*} b \varepsilon$ 'bom' (Kgg ${ }^{n} b \varepsilon$ 'fazer diariamente, ligeiro, gostar de fazer', Ing $\left.{ }^{n} b \varepsilon^{41}\right) \sim \mathrm{PJA}{ }^{* n} b \varepsilon c^{\curvearrowright}$;

\footnotetext{
${ }^{37}$ AM2 kuná 'escuridão'.

${ }^{38}$ AM2 buí.

${ }^{39}$ AM1 kundá=luné 'grão de milho', AM2 kendá=luné 'esmagar milho', AM3 lu-tenderi 'farinha', VO1 kunda=lun 'milho socado'.

${ }^{40}$ AM1 chuma=chú, AM2 chuma=njú.

${ }^{41}$ AM2 beé 'bom, bonito'; bé 'direito, correto'; beebe-tu-má 'mau, feio'; beebe-to-ndé 'mulher linda'; bea 'curar', VO1 ro=mbe 'dia'.
} 


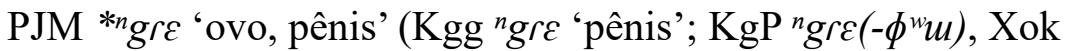
${ }^{n} g l \varepsilon$, Ing $={ }^{n} g r \varepsilon \varepsilon^{42}$ 'vagina') $\sim$ PJA *n $g r \varepsilon$ 'ovo, vagina';

PJM *ngredn 'dançar, cantar' (Kgg 'gred ${ }^{n}$ 'ensinar a dançar', $\mathrm{KgP} \tilde{w} \tilde{a}{ }^{n} g r \varepsilon d^{n}$ 'cantar', Xok $\tilde{v} \tilde{\varepsilon} \tilde{n}={ }^{n} g l \varepsilon d^{n}$ 'festejar, dançar, cantar', Ing $\widehat{d z} e=k r a j{ }^{43}$ 'dançar' (?)) PJA *n $g r \varepsilon(N)$;

PJM * ${ }_{r \varepsilon}$ 'deixar, abandonar' (Kgg ${ }^{2} \varepsilon$, Xok $\left.l \varepsilon-f^{n} / l \varepsilon-g^{n}\right) \sim$ PJA $*_{r \varepsilon}(N)$;

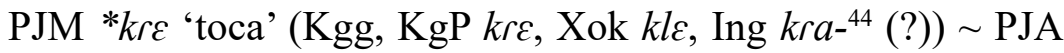
*kre 'buraco, toca, orifício'.

No seguinte caso a vogal parece ter sido afetada pela apofonia: PJM * $t_{\wedge} k a d^{n}$

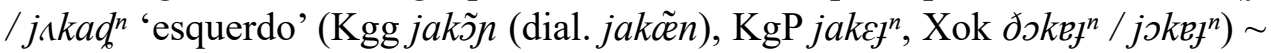
PJA *ke.

7.9. PJ *e não teria se mudado em PJM, ditongando-se para *je em ProtoJê Norocidental em sílabas com os seguintes ataques: ${ }^{*} b,{ }^{* n} g$, ${ }^{*} k r\left({ }^{*} k r j->\right.$ $*_{k j-}$. Em sílabas com os demais onsets $\left(*_{c}, *_{j}, *_{r}, *_{t},{ }^{*} p\right)$ permaneceu $*_{e}$, palatalizando as consoantes precedentes $\left({ }^{* n} d>*_{n}, *_{j}>*_{f}\right)$. A correspondência Maxakalí é $i$, enquanto em PJab se observa $*_{\varepsilon}$ ou $*_{i}$ :

$\mathrm{PJ} * k r e$ 'coxa' $\sim \mathrm{PJab} * k r \varepsilon$;

$\mathrm{PJ} *=c ̧ e / *=j e$ 'fio, corda' $\sim \mathrm{Mxk}=\int i t \sim \mathrm{PJab} * \widehat{d} \varepsilon^{45}$ 'fio, linha';

PJ *çare / *jare 'raiz' $\sim$ Maxakalí mĩm= jūp= fatit $\sim$ PJab *fari / $*=\widehat{d z}$ ari.

PJ *=pe 'lavar' Maxakalí $p i j \sim$ PJab *pi;

PJ *re 'dois' Maxakalí tij $\sim$ PJab */eri (?);

$\mathrm{PJ} *$ te "carrapato' $\sim$ Maxakalí tit $\sim$ PJab *tfitfi-ka (?);

$\mathrm{PJ} * k u=p e$ 'tocar, mexer' $\sim$ Maxakalí $\tilde{a}=p i t / \int \hat{i}=p i t$ 'tocar, pôr a mão';

PJ *pe(j) 'flatular' PJab *pij;

Provavelmente PJ *kret $~ * n g r e t^{2}$ 'gafanhoto-de-asa-vermelha' $\sim$ Maxakalí kidihit / kidit $t^{46}$ 'gafanhoto'.

\footnotetext{
${ }^{42} \mathrm{AM} 1$ an=an $(=)$ gré.

${ }^{43}$ AM1 adje=karai-pa, AM2 dikraia 'dançar'; dikreié 'baile', AM3 dje=kereia.

${ }^{44}$ AM2 krá-to-no 'tateto na toca'.

${ }^{45}$ Esta reconstrução parte da premissa que Arikapú $r \varepsilon$ é um empréstimo do Djeoromitxí; caso contrário, deveria-se reconstruir PJab * ${ }_{r \varepsilon}$ (reconstrução incompatível com os dados Jê e Maxakalí).

${ }^{46}$ Como a palavra Jê só é conservada no ramo Jê Central que não preserva a distinção entre $\mathrm{PJ} * e$ e $* i$, seria possível reconstruir PJ ${ }^{*} k r i t^{2}$ ou ${ }^{* n}$ grit ${ }^{2}$. Neste caso a forma corresponderia à forma curta em Maxakalí.
} 
No seguinte caso a vogal foi ainda nasalizada em Maxakalí:

PJ *pec 'fazer' Maxakalí mĩ̄.

Vejam-se alguns exemplos:

PJM *ku=jej 'veia' (Kgg, Xok $k u=j e j$ ', Ing $=g u=j e 47) \sim$ PJA

*kujek;

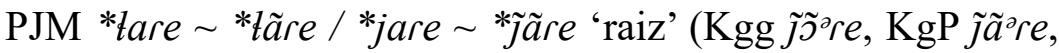

Xok jele $\sim j \tilde{e} l e$, ðele-dn 'enraizar') $\sim \mathrm{PJA} *$ çare / *jare;

$\mathrm{PJM} *=t e$ 'fio, corda, linha', *te-dn 'fiar' (Kgg $\tilde{w} \tilde{s}=\phi e, \phi \varepsilon-d^{n}$,

Xok $v \boldsymbol{v}=l e \sim \tilde{v} \tilde{\boldsymbol{\varepsilon}}=l e, \partial \varepsilon d^{n}$, Ing $=l e^{48}$ 'linha de pesca') $\sim \mathrm{PJA} *=c ̧ e$

$I^{*}=\jmath e$ 'fio, corda, arco';

PJM *kre 'coxa' (Kgg, KgP kre, Xok kle, Ing kre 49 'quadril') PJA *kje;

PJM *nge 'entrar.PL' (Kgg nge, Xok $\left.k e=g^{n} g e\right) \sim \mathrm{PJA} *={ }^{*} g j e(C)$;

PJM *jıgte 'ninho' (Kgg, KgP jagфe) PJA *çaçe / *jaçe;

PJM *pej ‘flatular' $\left(\right.$ Kgg $\left.p e j^{\curvearrowright}\right) \sim$ PJA *pe $e^{50}$.

No seguinte caso a vogal em PJM foi afetada pela apofonia:

PJM ${ }^{* n} b \varepsilon d^{n}$ 'esposo' (Kgg, KgP, Xok $\left.{ }^{n} b \varepsilon d^{n}\right) \sim \mathrm{PJA}{ }^{* n} b j e d^{n}$.

7.10. PJ *i não teria se mudado em nenhum dos ramos. Em Maxakalí são encontradas as correspondências $\varepsilon$ (na posição tônica) e $\dot{t}$ (na posição átona); em PJab a correspondência é $* i$ :

PJ *çi / *ji 'colocar' Maxakalí $\int \varepsilon j$ 'guardar, tomar conta' PJab *ji;

$\mathrm{PJ} * c ̧ i / *_{j i}$ 'osso' $\sim \mathrm{PJab} *(j) i$

$\mathrm{PJ} * c ̧ \tilde{\imath}=j i / *^{*} \tilde{\imath}=j i(\sim-i-)$ 'nome' $\sim$ Maxakalí $/ \hat{t}=\int \varepsilon t / \tilde{a}=\int \varepsilon t \sim \mathrm{PJab}$ *tãfi * *afi;

PJ *piji 'um' Maxakalí pifect;

talvez PJ *pire 'pesado' Maxakalí pitijj.

\footnotetext{
${ }^{47} \mathrm{VO} 3$ nangâu $=$ guyé 'pulso', talvez VO3 mié 'veia'.

${ }^{48} \mathrm{AM} 1 u a=l e ́, \mathrm{AM} 2$ rei=lé, $\mathrm{AM} 3 d o=l e ́$.

${ }^{49} \mathrm{AM} 2$ ap $=k r e ́$.

${ }^{50}$ Contra (Ribeiro \& Voort 2010: 555), *-k não faz parte desta raiz verbal (cf. Apinajé $i t=p e$, Xavánte $p i / p i$-ri), sendo um sufixo nominalizador na palavra derivada *pe- $k$ 'flatulência' (Pykobjê pik), cf. PJA *tu 'morrer' e *tu-k 'morto'.
} 
Maxakalí tit 'mãe, irmã da mãe, animal de grande porte' poderia ser comparado com Proto-Jê Norocidental *n $d i$ 'mulher', mas preferimos comparar este vocábulo com PJM *tr 'mulher' $(<\mathrm{PJ} * t \gamma)$ por causa da correspondência vocálica.

Vejam-se alguns exemplos da família Jê:

PJM * $* i / * j i$ 'deitar, colocar deitado' $(\operatorname{Kgg} \phi i$, Xok $\partial i / j i) \sim$ PJA *çi / *ji 'colocar';

$\mathrm{PJM} * *_{i=j i} / * j i=j i \sim-u-\sim-u$ 'nome' $(\operatorname{Kgg} j i j i \sim j i j i \sim j i j j, \mathrm{KgP}$ $j i j i \sim j u j u$, Xok $\partial i j j i j / j i j i) \sim \operatorname{PJA} c ̧ \tilde{l}=j i / * \tilde{j} \tilde{i}=j i(\sim-i-)$.

PJM *pir ${ }^{\curvearrowright}$ 'um, pouco' (Kgg, KgP pir ${ }^{\curvearrowright}$, Xok pil’, Ing bi- $\left.{ }^{51}\right)$ provavelmente provém de $\mathrm{PJ} *$ *iji, mas a consoante em coda, assim como a ausência da segunda sílaba, permanecem inexplicadas.

7.12. PJ * $\tilde{j}$ teria se preservado em PJA, sofrendo alteamento em PJM $(* \tilde{\jmath}>$ $* \tilde{u})$. A correspondência na língua Maxakalí é $\tilde{o}$; em PJab encontra-se * $\tilde{u}(* \tilde{z}$ em um morfema gramatical):

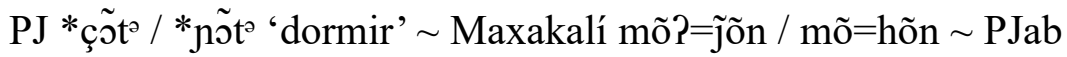
*nũtã;

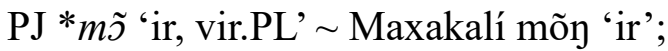

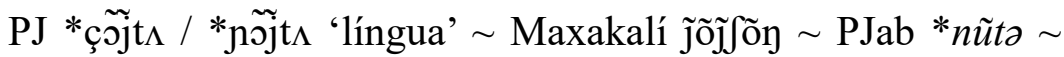
${ }^{* n}$ dutz;

$\mathrm{PJ}$ *ç̃̃ / *ñ̃ ‘dar' Maxakalí hõm 'dar, pagar. SG' PJab *2ũ;

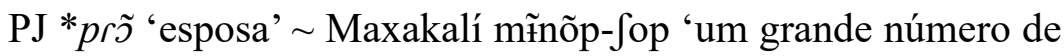
gente da mesma categoria';

$\mathrm{PJ} * p \tilde{s}$ 'lavar' $\sim \mathrm{PJab} * p \tilde{z} \sim * p \tilde{u}$

$\mathrm{PJ} * t \tilde{\partial}$ 'negação' $\sim \mathrm{PJab} * t \tilde{\partial}$.

Vejam-se alguns exemplos:

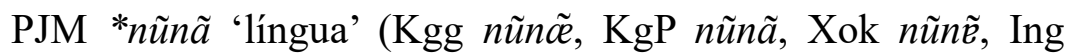
$\left.n u^{n} d a^{52}\right) \sim \mathrm{PJA} * c ̧ \tilde{\jmath} \tilde{j} t \Lambda / * n \tilde{\partial} \tilde{j} t \Lambda ;$

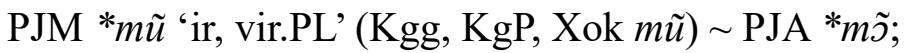

PJM *pr̃ũ 'esposa' (Kgg, KgP pr̃u, Xok pĩ ũ, Ing pro ${ }^{53}$ 'marido')

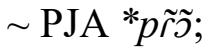

\footnotetext{
${ }^{51}$ AM2 bipá, AM3 biré.

${ }^{52} \mathrm{AM} 1$ agn=upá (?), AM2 am=undá, VO1 nomdá, VO3 nâurá (?).

${ }^{53} \mathrm{VO} 1$ pró.
} 


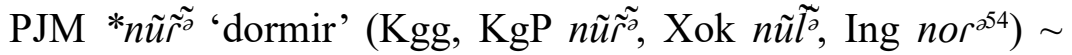
PJA *ç̃̃t / *ñ̃t;

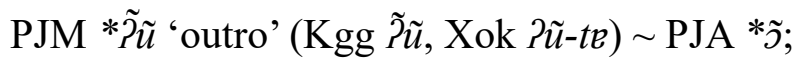

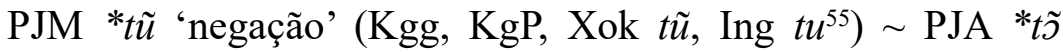
'proibitivo'.

Nos seguintes casos, apesar de a palavra PJA conter $* \tilde{y}$, os cognatos em PJM, Maxakalí ou PJab apresentam uma vogal não labial:

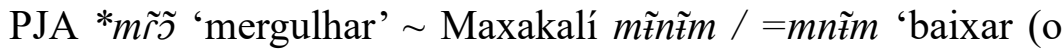
anzol para pescar)' PJab *mrz̃ 'dar banho, banhar, limpar';

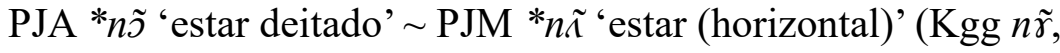
$\mathrm{KgP} n \tilde{\partial}$, Xok $n \tilde{\partial}$, Ing $\left.n a_{-}{ }^{56}\right) \sim \mathrm{PJab} * n \tilde{\text { ' }}$ copular';

$\mathrm{PJA} * b \tilde{s}$ 'capim, grama' $\sim \mathrm{PJM} *=p \tilde{\kappa}$ 'roça' $(\operatorname{Kgg} \tilde{P} \tilde{c}=p \tilde{\gamma} / j a=p \tilde{\gamma}$, $\operatorname{KgP}(j a=) p \tilde{\partial}$, Xok $2 \tilde{\varepsilon}=p \tilde{\jmath} / j \jmath=p s)$; nesse caso a correspondência consonantal é irregular.

Provavelmente nessas palavras na língua Proto-Jê havia um fonema diferente de $* \tilde{y}$, mas não é possível tirar uma conclusão decisiva baseando-se nestes dados devido à escassez de exemplos.

7.13. PJ $* \tilde{\imath}$ teria se conservado nos dois ramos, sofrendo um alteamento em $\operatorname{PJM}\left({ }^{*} \tilde{\imath}>*^{*}\right)$ próximo a consoantes palatais. As correspondências nas línguas Maxakalí e PJab não parecem ser sistemáticas e devem ainda ser estudadas:

PJ *prĩm 'fome' Maxakalí pittp 'querer, desejar';

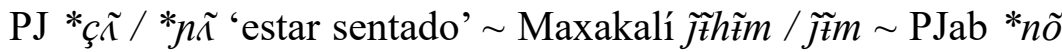
'sentar, pousar';

PJ *kaỹ̃ 'cobra' Maxakalí kãjã

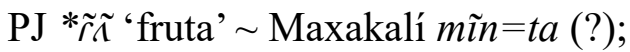

PJ *krĩ̃ 'montanha' Maxakalí jĩktij 'morro, montanha, serra' (?).

Vejam-se alguns exemplos:

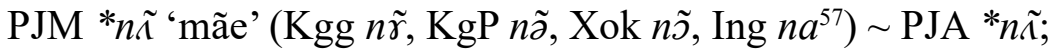

\footnotetext{
${ }^{54} \mathrm{AM} 1$ ani=noron 'dormir', AM2 nóro 'ter sono', $i=$ non-gran 'dormir, vou dormir', VO1 nor, noró 'dormir', nor-ján 'cama'.

${ }^{55} \mathrm{AM} 1$ djachike=tu-ma 'não querer', $\mathrm{AM} 2$ dada=tu-de 'não chove', alaa=tu- $k u a ́$ 'não chore', djibre=tu-má 'não entender'.

${ }^{56}$ AM2 náma 'deitar-se'.

${ }^{57}$ AM2 am=ná-kullá 'mulher', VO3 ná 'mãe'.
} 


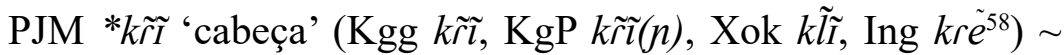
PJA *krĩy;

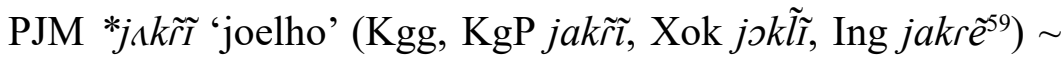
PJA *çikrĩ̃j;

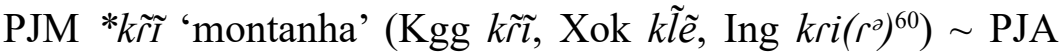
$* k r \tilde{y}$;

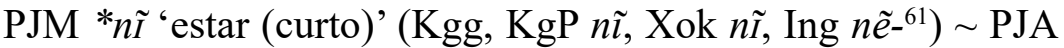
*çَ̃ / $n \tilde{\Lambda}$ 'estar sentado'.

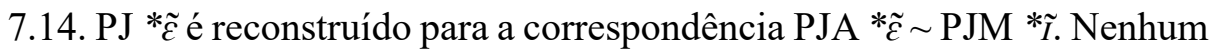
cognato foi encontrado nas línguas Maxakalí ou Jabutí para nenhuma palavra com esta correspondência. Vejam-se os exemplos:

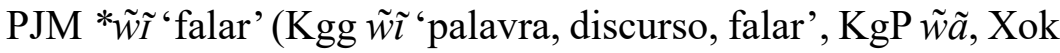
$\tilde{v} \tilde{I}) \sim \mathrm{PJA} * b \tilde{\varepsilon}$;

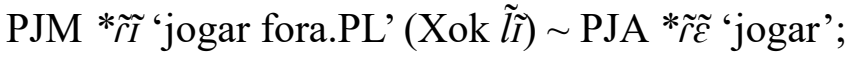

$\mathrm{PJM} * t \tilde{I}$ 'ir, vir.SG' $\left(\mathrm{Kgg}, \mathrm{KgP} t \tilde{\imath}\right.$, Xok $t \tilde{I}$, Ing $\left.t e^{62}\right) \sim \mathrm{PJA} * t \tilde{\varepsilon}$ 'ir. SG'.

7.15. PJ *ĩ não teria se modificado em PJA, sendo $*_{I}$ o reflexo em PJM. Corresponde regularmente à mesma vogal em Maxakalí e em PJab:

PJ *pĩm 'madeira, lenha' Maxakalí mĩhĩm / mĩm $\sim$ PJab *pi-țja 'fogo';

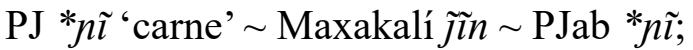

$\mathrm{PJ} * n \tilde{\imath}$ 'espinho' $\sim \mathrm{PJab} * n \tilde{i}$;

PJ *çĩ $(p)$ - / nĩ $(p)$ - 'prefixo de partes de corpo' $\sim$ Maxakalí $\tilde{\jmath} \mathfrak{l}(p / m)-\sim \operatorname{PJab} * n \tilde{l}-$.

No seguinte caso provavelmente houve monotongação na família Jê: PJ *mĩ ‘jacaré' Maxakalí mã̃ã̃j.

Vejam-se alguns exemplos:

\footnotetext{
${ }^{58}$ AM2 aut=krein, AM3 kiri-nidjá 'gorro', chiri-nguaré 'lenço', VO1 krí, krĕ, VO3 kré.

${ }^{59} \mathrm{AM} 1$ am=itkrin, $\mathrm{AM} 2$ am=iekré, $\mathrm{VO} 3$ =nakaré.

${ }^{60} \mathrm{AM} 1$ kériri 'alto', AM2 keriri, VO1 kerý, kererý.

${ }^{61} \mathrm{AM} 2$ ne-má 'sentar-se', ne(n)-ma 'senta aqui', tan=grau=ne-ma 'sentar-se no solo'.

${ }^{62}$ AM2 ara te mo 'onde tu vais?'.
} 
PJM *nĩj^ 'fumaça' (Kgg nĩja, Xok nĩjo, Ing pi=nije $\left.{ }^{63}\right) \sim$ PJA *çijı / *nij $\Lambda$;

PJM *pĩ 'fogo, lenha' (Kgg $p \tilde{\imath}, \operatorname{KgP} p \tilde{\imath}(n)$, Xok pĩ , Ing $\left.p e^{64}\right) \sim$ PJA *pĩm 'madeira, lenha';

PJM *nĨ 'carne' (Kgg, $\mathrm{KgP} n \tilde{\imath}$, Xok $n \tilde{I}$, Ing $\left.{ }^{n} d u=n e^{65}\right) \sim \mathrm{PJA} * n \tilde{i}$;

PJM *nĨ- 'prefixo de partes de corpo' $(\mathrm{Kgg}, \mathrm{KgP} n \tilde{l}$, Xok $n \tilde{I}) \sim$ PJA *nĩ $(p)$-.

7.16. Provavelmente existiam ainda outras vogais em Proto-Jê. Eis alguns exemplos de casos que não se encaixam nas regras descritas acima:

PJM *lcj 'folha' (Kgg, KgP $\phi \varepsilon^{j}$, Xok $\left.\delta \varepsilon j^{2}\right) \sim$ PJA *çoj (esta palavra não é afetada pela apofonia em PJM, o que normalmente ocorre em raízes, cujos cognatos em PJA apresentam uma oclusiva na posição de coda);

PJM *nĩjı 'fumaça' (Kgg nĩja, Xok nĩjo, Ing pi=nije) PJA

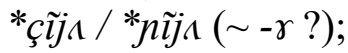

PJM * $t_{\Lambda}$ 'amargo' $\left(\mathrm{Kgg}, \mathrm{KgP} \phi^{w} a\right.$, Xok $\left.っ\right) \sim \mathrm{PJA} * * \gamma$;

$\mathrm{PJM} * r e$ 'descer' (Kgg, KgP əre, Xok le) PJA *rwr(C) (esta palavra não é afetada pela apofonia em PJM, o que normalmente ocorre em raízes, cujos cognatos em PJA apresentam uma oclusiva na posição de coda);

PJM *prs 'morder' (Kgg, KgP pra, Xok pls, Ing $\left.p r a^{66}\right) \sim$ PJA pro (apenas Xerénte $p r o$ 'comer carne');

PJM *ngra 'assado no forno, na brasa' (Kgg $y \tilde{r} \tilde{y}$, Xok ${ }^{n} g l e$, Ing $\left.{ }^{n} \mathrm{grau}^{67}\right) \sim$ PJA *n gro 'queimar, assar no fogo';

\footnotetext{
${ }^{63} \mathrm{AM} 2$ pi=nie, $\mathrm{VO} 1$ pei=gyje, $\mathrm{VO} 3$ pi=niě.

${ }^{64} \mathrm{AM} 1$ pein 'fogo'; pin-grá 'brasa, chama'; pui-teichó 'fumaça', AM2 péin 'fogo'; pin-grá 'brasa, chama'; pim-pó 'lenha'; pet-krén, pet-kren 'fazer fogo'; pen-güité 'buscar lenha'; pi-nie 'fumaça', AM4 pén 'fogo'; pin-coró 'lenha'; pi-ná 'fumaça', VO1 péi 'fogo'; pingrá 'chama'; pei-gyje 'fumaça', VO3 pái 'fogo'; pin-grá 'chama'; pi-niě 'fumaça', LI npai 'fuego'.

${ }^{65}$ AM1 ndeiné, AM2 nduiné 'carne'; doin-dangüangüe 'caçar'; i=toin tan ramá ‘vou caçar'; doiro jámo, ndoirojámo 'carnear'; peten guten doiro jamo 'venha carnear'; ajo dain dain jámo 'vamos juntos caçar bichos', VO3 dyné.

${ }^{66} \mathrm{AM} 3$ it=pirai-má, VO1 pràn, VO3 ak=pyreá (?).

${ }^{67}$ AM2 graun 'carne cozida'; gro-to-hó 'carne crua'; dau=gró 'milho assado'; re=nde=ngron 'caraguatá assado', VO1 kunda=grón 'milho assado'; grein=grōn 'caraguatá assado', rea=ngràn 'milho assado'; rai=ngrāni 'caraguatá assado'.
} 


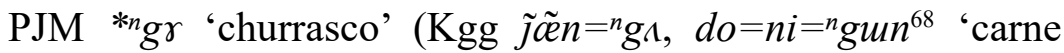
assada') PJA *n $g a$ 'assar';

PJM *ngoj ‘água, rio' (Kgg, KgP, Xok $\left.{ }^{n} g o j^{\curvearrowright}\right) \sim$ PJA *ngoj 'água (parada)' ou *ngmj 'água (corrente)';

PJM * $\star_{\Lambda} / *_{j \varsigma}$ 'perna' $\left(\operatorname{Kgg} \phi^{w} a\right.$, Xok $\left.\partial / j o\right) \sim$ PJA *çaj 'coxa, perna' (esta palavra não é afetada pela apofonia em PJM, o que normalmente ocorre em raízes, cujos cognatos em PJA apresentam uma oclusiva na posição de coda).

\section{Bloqueamento de apofonia}

Sugerimos que a ocorrência da apofonia em PJM teria sido bloqueada pela presença de uma consoante oclusiva na posição de coda em Proto-Jê (daqui em diante, marcaremos as raízes reconstruídas não suscetíveis à apofonia com o símbolo ? no final da sílaba e as raízes suscetíveis à mesma com \#.):

PJM * $k a=k \wedge$ ? 'vento' $<\mathrm{PJ} *(k a=) k o k^{2}$;

PJM *k^? 'mosquito, borrachudo' < PJ kop 'mosquito ou mosca'.

As raízes PJM suscetíveis a apofonia provêm de raízes que não terminavam em oclusivas em PJ:

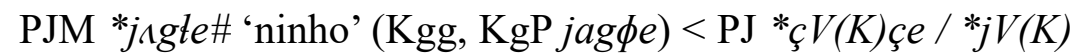
çe;

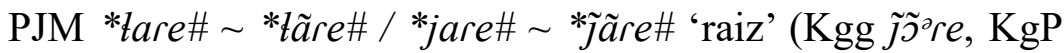

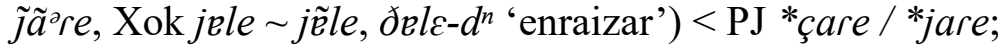

PJM *=le\# 'fio, linha', * $\varepsilon_{\varepsilon}-d^{n}$ 'fiar' $\left(\operatorname{Kgg} \tilde{w} \tilde{s}=\phi e, \phi \varepsilon-d^{n}\right.$, Xok $v \varepsilon=l e \sim \tilde{v} \tilde{B}=l e, \partial \varepsilon d^{n}$, Ing =le 'linha de pesca') $<\mathrm{PJ} *=c ̧ e / *=j e$ 'fio, corda';

$\mathrm{PJM} * t_{o \#} / *^{*}=j o \#$ 'pus' $\left(\mathrm{Kgg} \phi^{w} O, \tilde{j} \tilde{a} n=j o\right.$ 'látex, resina', $\mathrm{KgP}$ $\tilde{j} \tilde{a}=n \tilde{u}$ 'látex, resina', Xok $о$ ) $<\mathrm{PJ} * c ̧ u / * j u$;

PJM *ngrc\# 'ovo, pênis' (Kgg ' $g r \varepsilon$ 'pênis'; KgP ${ }^{n} g r \varepsilon\left(-\phi^{w} w\right)$, Xok

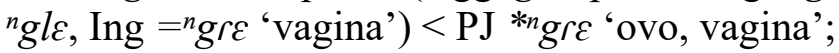

PJM * * $r$ \# 'deixar, abandonar' (Kgg ${ }^{\curvearrowright} \varepsilon \varepsilon$, Xok $\left.l \varepsilon-f^{n} / l \varepsilon-g^{n}\right)<$ PJ $*_{r \varepsilon}(N)$;

PJM *ka\# 'árvore' (Kgg, KgP ka, Xok ks) < PJ *ko 'pé de arvore, chifre, borduna';

${ }^{68} \mathrm{VO} 3 \mathrm{do}=$ ni=ngýn. 
PJM * $t_{\Lambda} \#$ 'mamar' $\left(\operatorname{Kgg}\right.$ pag $=\phi^{w} a$, pl. pig $=\phi^{w} a, \operatorname{KgP} p a=\phi^{w} a$, Xok $p \varepsilon\left(g^{n}\right)=$ ð) < PJA *ço 'chupar';

PJM *ng^\# 'piolho, caruncho' (Kgg nga 'piolho, caruncho, coró', $\mathrm{KgP}{ }^{n} g a$, Xok ${ }^{n} g$, Ing $\left.{ }^{n} g a\right)<\mathrm{PJ}$ *ngo 'piolho';

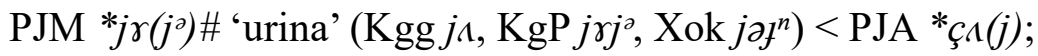

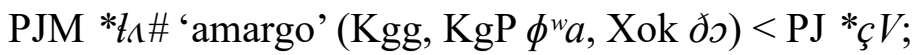

PJM *nĩjı\# 'fumaça' (Kgg nĩja, Xok nĩjo, Ing pi=nije) < PJ *çĩjV/*nĩjV;

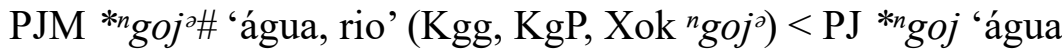
(parada)' ou *nguj 'água (corrente)'.

Em raízes não derivadas terminadas em uma coda pós-nasalizada tanto em PJ quanto em PJM ocorre o grau B das vogais em todos os casos conhecidos:

PJ *tug ${ }^{n a}$ 'seco'> PJM *tog ${ }^{n}$ 'seco (de plantas)' (Kgg, KgP tog ${ }^{n}$, Xok $\operatorname{tug}^{n}$ );

PJ *n $b e d^{n a}$ 'esposo'> PJM *n $b \varepsilon d^{n}\left(\mathrm{Kgg}, \mathrm{KgP}, \mathrm{Xok}^{n} b \varepsilon d^{n}\right)$.

Poder-se-ia pensar que a realização fonológica do sufixo ativizador depende da qualidade da consoante final do Proto-Jê, mas tal relação, se existente, não é óbvia:

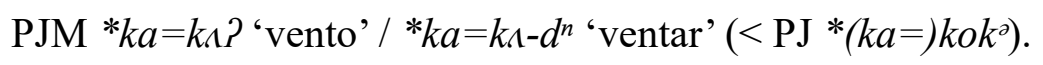

As raízes $\mathrm{PJM}$ não suscetíveis a apofonia podem se combinar com qualquer dos alomorfes do sufixo ativizador:

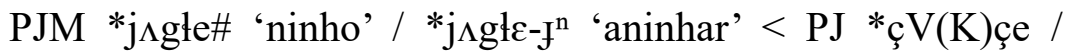
*j $\mathrm{V}(\mathrm{K})$ çe;

PJM *koksє\# 'podre' / *kokra-jn 'apodrecer' < PJ *kroj (?);

PJM *=le\# 'fio, corda, linha' / *1e-dn 'fiar' < PJ *=çe / *=je 'fio, corda';

PJM *ng ${ }_{\Lambda} \#$ 'piolho, caruncho' / *nga-d n 'criar piolho, carunchar'

$<$ PJ *ngo 'piolho';

PJM *1ム\# 'amargo' / *1a-gn 'amargar' < PJ *çV;

PJM *nĨj $\Lambda$ 'fumaça' / *nĩja-gn 'fazer fumaça' < PJ *nĩjV;

PJM * Ło\# / *=jo\# 'pus' / *ło-b ' 'criar pus, inflamar-se' < PJ *çu / *ju. 
Talvez a escolha do alomorfe dependa da qualidade da vogal tônica, mas por enquanto nenhuma regra pode ser formulada.

\section{Considerações finais}

Neste trabalho procuramos demonstrar uma hipótese reconstrutiva do sistema vocálico do Proto-Jê Meridional diferente das propostas por Jolkesky (2010) e D'Angelis (2008). A diferença principal consiste no tratamento de correspondências por meio das quais esses autores derivam $* a, * \tilde{a}$ e $* \tilde{\varepsilon}$. Advogamos pela hipótese do desenvolvimento de protofonemas como $*_{\Lambda}(<\mathrm{PJ}$ $\left.*_{o}\right),{ }^{*} a\left(<\mathrm{PJ} *_{0}\right) \mathrm{e} * \tilde{a}\left(<\mathrm{PJ} * a\right.$ e $\left.*_{1}\right)$ respectivamente, baseando-nos em algumas idiossincrasias registradas sincronicamente nas línguas Jê Meridionais. É fácil ver ainda que a distância fonética entre estas vogais em PJM e suas fontes PJ é mais curta, se aceitarmos a presente reconstrução e não as de Jolkesky e de D’Angelis. Desse modo, a vogal PJ *a (e PJ $\left.*_{\curlywedge}\right)$ teria se nasalizado em PJM e PJ * teria ocupado o seu espaço. Em Kaingáng, a vogal PJM *a $(<\mathrm{PJ} *)$ teria se nasalizado mais uma vez, sendo o espaço dela ocupado por PJM $*_{\Lambda}$; a

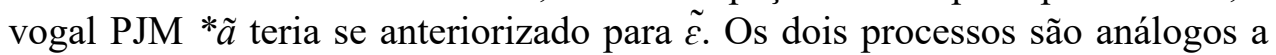
um processo que ocorreu na história da língua Tapirapé (Tupí-Guaraní, tronco Tupí): $\mathrm{PTG} *_{o}>$ Tapirapé $a$, PTG $* a>$ Tapirapé $\tilde{a}$, PTG $* \tilde{a}>$ Tapirapé $\tilde{i}$ (Soares \& Leite 1991), assim como nas línguas Parakanã e Asuriní do Tocantins e nas línguas do Subramo V - Araweté, Anambá e Asuriní do Xingu (Rodrigues e Cabral 2001:33).

A solução adotada neste trabalho é corroborada pelo tratamento de empréstimos de uma língua Tupí-Guaraní (possivelmente do Mbyá) encontrados na língua Kaingáng:

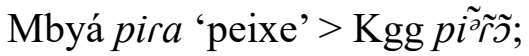

$$
\begin{aligned}
& \text { Mbyá tupã 'deidade' > Kgg topẽe; } \\
& \text { Mbyá pari ‘cercado, curral para apanhar peixes'> Kgg p } \tilde{\jmath}^{\curvearrowright} r i \text {. }
\end{aligned}
$$

A reconstrução do sistema fonológico do PJM deve contribuir para os estudos comparativos de todo o tronco Macro-Jê e para a tipologia de mudanças fonéticas e fonológicas.

\section{Referências}

Aikio, Ante (Luobbal Sámmol Sámmol Ánte). 2015. Notes on the development of some consonant clusters in Hungarian. Peri orthotētos etymōn. Uusiutuva uralilainen etymologia (Sampsa Holopainen, Janne Saarikivi (eds.)). Uralica Helsingiensia. (No prelo.)

Amado, Rosane de Sá. 2004. Aspectos morfofonológicos do Gavião-Pykobjê. São Paulo: Universidade de São Paulo. Tese de Doutorado. 
Ambrosetti, Juan Bautista. 1896. Materiales para el estudio de las lenguas del grupo Kaingangue (Alto Paraná). Boletín de la Academia Nacional de Ciencias de Córdoba, 14:331-382.

Bubrix, D. V. (Бубрих, Д. В.) 1948. Istoričeskaja fonetika udmurtskogo jazyka (Историческая фонетика удмуртского языка). Iževsk: Udmurtgosizdat.

Camargo, Nayara da Silva. 2010. Língua Tapayúna: aspectos sociolingüísticos e uma análise fonológica preliminar. Campinas: UNICAMP. Dissertação de Mestrado.

Carlo Sandro de Oliveira Campos. 2009. Morfofonêmica e morfossintaxe do Maxakalí. Belo Horizonte: UFMG. Tese de Doutorado.

Cavalcante, Marita Pôrto. 1987. Fonologia e morfologia da língua Kaingáng: o dialeto de São Paulo comparado com o do Paraná. Campinas: UNICAMP. Tese de Doutorado.

Costa, Consuelo de Paiva Godinho. 2007. Apyngwa rupigwa: nasalização em Nhandewa-Guarani. Campinas: UNICAMP. Tese de Doutorado.

D’Angelis, Wilmar R. 2008. Pensar o Proto-Jê Meridional e revisitar o Proto-Jê, numa abordagem pragueana. Relatório Acadêmico de Estágio Pós-Doutoral em Linguística Histórica. Brasília: Universidade de Brasília.

D’Angelis, Wilmar R. 2009. O Xokléng, o Kaingáng e sua filiação ao Proto-Jê: antecedentes para uma reflexão atual. Revista Brasileira de Linguística Antropológica 1(2):117-148.

Davis, Irvine. 1966. Comparative Jê phonology. Estudos Lingüísticos: Revista Brasileira de Lingüística Teórica e Aplicada 1(2):20-24.

Epps, Patience. 2005. A Grammar of Hup. University of Virginia. Tese de Doutorado.

Grannier, Daniele Marcelle. 2009. Estrutura silábica e nasalização em Akwẽ-Xerente. Signótica 21:245-260. Goiânia.

Jolkesky, Marcelo Pinho De Valhery. 2009. Fonologia e prosódia do Kaingang falado em Cacique Doble (RS). Anais do XIV SETA - Seminário de Teses em Andamento 3:675-685. Campinas: Editora do IEL - UNICAMP.

Jolkesky, Marcelo Pinho De Valhery. 2010. Reconstrução fonológica e lexical do ProtoJê Meridional. Campinas: UNICAMP. Dissertação de Mestrado.

Kassian, Alexei; George Starostin; Anna Dybo; Vasiliy Chernov. 2010. The Swadesh wordlist. An attempt at semantic specification. Journal of Language Relationship $4: 46-89$.

Lista, Ramón. 1883. El territorio de las misiones. Buenos Aires: La Universidad.

Mattos, Rinaldo de. 1973. Fonêmica xerente. Série lingüística 1. Brasília: SIL.

Nikolaeva, Irina 2006. A Historical Dictionary of Yukaghir. Trends in Linguistics Documentation 25. Berlin \& New York: Mouton de Gruyter.

Nikulin, Andrey. 2015. On the genetic unity of Jê-Tupí-Karib (Верификация гипотезы о же-тупи-карибском генетическом единстве). Moscou: Universidade Estatal de Moscou. Dissertação de conclusão do curso. 
Nikulin, Andrey. 2016. Phonological and lexical reconstruction of Proto-Jê. (No prelo.)

Oliveira, Christiane Cunha de. 2005. The language of the Apinajé people of Central Brazil. University of Oregon. Tese de Doutorado.

Picanço, Gessiane Lobato. 2005. Mundurukú: phonetics, phonology, synchrony, diachrony. University of British Columbia. Tese de Doutorado.

Pickering, William Alfred. 2010. A fonologia Xavante: uma revisitação. Campinas: UNICAMP. Dissertação de Mestrado.

Popjes, Jack; Josephine Popjes. 1971 [2009]. Phonemic statement of Canela. Preliminary version. Anápolis: Associação Internacional de Linguística - SIL Brasil.

Ribeiro, Eduardo Rivail e Hein van der Voort. 2010. Nimuendajú was right: the inclusion of the Jabutí language family in the Macro-Jê stock. IJAL 76(4): 517-570.

Rodrigues, Aryon Dall'Igna. 1981. Abertura e ressonância. Estudos Linguísticos. Anais dos Seminários do GEL 4:324-333.

Rodrigues, Aryon Dall'Igna. 2012. O Xokléng, o Kaingáng e sua filiação ao ProtoJê: antecedentes para uma reflexão atual. Revista Brasileira de Linguística Antropológica 4(2):279-288.

Rodrigues, Aryon Dall'Igna e Ana Suelly A. C. Cabral. 2002. Revendo a classificação interna da família Tupí-Guaraní. Línguas indígenas brasileiras: fonologia, gramática, história (Ana Suelly A. C. Cabral \& Aryon D. Rodrigues (orgs.)) 1:327337. Belém: EDUFPA.

Sá, Rosane Muñoz de. 1999. Análise fonológica preliminar do Pykobjê. São Paulo: USP. Dissertação de Mestrado.

Soares, Marília Facó e Yonne Leite. 1991. Vowel shift in the Tupi-Guarani language family: a typological approach. South American Indian languages (Mary Ritchie Key, ed.), 36-53. Philadelphia: University of Pennsylvania Press.

Souza, Shelton Lima de. 2008. Descrição fonético-fonológica da língua AkwenXerente. Brasília, Universidade de Brasília. Tese de Doutorado.

Starostin, George. 2010. Preliminary lexicostatistics as a basis for language classification: A new approach. Journal of Language Relationship 3:79-116.

Stout, Mickey; Ruth Thomson. 1974. Fonêmica Txukuhamẽi (Kayapó). Série Lingüistica 3. Brasília: Summer Institute of Linguistics (SIL).

Thomas, Guillaume. 2014. A split analysis of nasal harmony in Mbya. Revista Linguística / Revista do Programa de Pós-Graduação em Linguística da Universidade Federal do Rio de Janeiro 10(2): 75-105.

Vogt, P. Fr. 1904. Die Indianer des Obern Paraná. Mitteilungen der Anthropologischen Gessellschaft in Wien, 34:200-221, 353-377. Wien.

Voort, Hein van der. 2007. Proto-Jabutí: um primeiro passo na reconstrução da língua ancestral dos Arikapú e Djeoromitxí. Bol. Mus. Para. Emillio Goeldi. Ciências Humanas 2(2): 133-168. Belém.

Wiesemann, Ursula. 1958. Notas sobre Proto-Kaingáng: um estudo de quatro dialetos. Tradução de Miriam Lemle. Rio de Janeiro: Summer Institute of Linguistics. 21p. m.s. 
Wiesemann, Ursula. 1978. Os dialetos da língua Kaingáng e o Xokléng. Arquivos de Anatomia e Antropologia 3:197-217. Rio de Janeiro: Instituto de Antropologia Professor Souza Marques.

Wiesemann, Ursula Gojtéj. 2011. Dicionário Kaingang-Português, PortuguêsKaingang. $2^{\mathrm{a}}$ edição atualizada pelo Novo Acordo Ortográfico. Curitiba: Esperança.

Data recebimento: $10 / 07 / 2015$

Data aceite: $12 / 08 / 2015$ 